PHYSICAL REVIEW D 93, 103518 (2016)

\title{
Spectrum of anomalous magnetohydrodynamics
}

\author{
Massimo Giovannini \\ Department of Physics, Theory Division, CERN, 1211 Geneva 23, Switzerland \\ and INFN, Section of Milan-Bicocca, 20126 Milan, Italy \\ (Received 7 September 2015; published 18 May 2016)
}

\begin{abstract}
The equations of anomalous magnetohydrodynamics describe an Abelian plasma where conduction and chiral currents are simultaneously present and constrained by the second law of thermodynamics. At high frequencies the magnetic currents play the leading role, and the spectrum is dominated by two-fluid effects. The system behaves instead as a single fluid in the low-frequency regime where the vortical currents induce potentially large hypermagnetic fields. After deriving the physical solutions of the generalized AppletonHartree equation, the corresponding dispersion relations are scrutinized and compared with the results valid for cold plasmas. Hypermagnetic knots and fluid vortices can be concurrently present at very low frequencies and suggest a qualitatively different dynamics of the hydromagnetic nonlinearities.
\end{abstract}

DOI: 10.1103/PhysRevD.93.103518

\section{INTRODUCTION}

Electrically conducting media are customarily described as a single fluid in the low-frequency branch of the plasma spectrum. This approach has been extensively applied to the analysis of hydromagnetic nonlinearities [1] evolving in terrestrial [2] and astrophysical plasmas [3-5]. The same strategy cannot be extended to higher frequencies where the one-fluid description is no longer tenable [6] and the plasma must be treated, at least, as a double fluid. This well-known aspect of conventional electromagnetic plasmas stems directly from the properties of the vector currents which are associated, in the high-frequency limit, with the ions and with the electrons. When the plasma is globally neutral the total vector current is instead Ohmic in the lowfrequency domain.

A problem of a similar nature occurs in anomalous magnetohydrodynamics [7] describing a charged fluid where axial and vector currents are simultaneously present: While the axial currents are not conserved because of the triangle anomaly, the vector currents are eventually Ohmic. The purpose of this investigation is a systematic discussion of the spectrum of anomalous magnetohydrodynamics (AMHD). The equations of AMHD differ from the ones where only chiral currents are present [8,9] at finite fermionic density. They generalize the system firstly explored in Ref. [10] accounting for the evolution of the hypermagnetic and hyperelectric fields in the electroweak plasma. Indeed, in the symmetric phase of the electroweak theory the nonscreened vector modes of the plasma correspond to the hypercharge which has a chiral coupling to

*massimo.giovannini@cern.ch

Published by the American Physical Society under the terms of the Creative Commons Attribution 3.0 License. Further distribution of this work must maintain attribution to the author(s) and the published article's title, journal citation, and DOI. fermions. The axial currents may be associated with the evolution of the chemical potential or with the presence of an axionlike field [11,12] (see also [13]). In both cases the plasma may host parity-odd configurations of the gauge fields characterized nonvanishing hypermagnetic gyrotropy $\vec{B} \cdot \vec{\nabla} \times \vec{B}$ which is the hydromagnetic analog of the kinetic gyrotropy (i.e. $\vec{v} \cdot \vec{\nabla} \times \vec{v}$ ) naturally appearing in the discussion of mean-field dynamos $[1,4]$. The dynamical production of hypermagnetic knots and Chern-Simons waves during inflation offers a potentially viable mechanism for the generation of the baryon asymmetry of the Universe (see last two papers in [10]). In AMHD the hypermagnetic current is complemented by a vortical current possibly leading to the formation of fluid vortices.

The same class of physical systems previously discussed in the electroweak plasma also arises in the framework of the so-called chiral magnetic effect [14]. Both phenomena are often presented as macroscopic manifestations of triangle anomalies. The model of the chiral liquid emerging in the context of AMHD could then be relevant also in the context of the chiral magnetic effect insofar as axial currents and quark vector currents are concurrently present in the strongly interacting plasma. In the absence of finite conductivity effects (see e.g. $[15,16]$ ) the validity of the second law of thermodynamics is guaranteed by the simultaneous presence of a hypermagnetic current and of a chiral vortical term. In AMHD the vector currents (eventually responsible of Ohmic dissipation), the chiral currents (determining the anomalous effects) and the vortical currents (required by the second principle of thermodynamics) are all described by the appropriate kinetic coefficients. Whenever possible AMHD is discussed in analogy with the spectrum of conventional plasmas. More specifically the plan of this investigation is the following. In Sec. II we discuss the relativistic problem and derive the general form of the kinetic coefficients. In Sec. III the properties of the 
two-fluid equations are analyzed while Sec. IV is devoted to the dispersion relations in the high-frequency domain. The one-fluid equations and their implications are presented in Sec. V. Section VI contains our concluding remarks. To avoid digressions some relevant technical aspects have been relegated to the Appendix.

\section{RELATIVISTIC FORMULATION AND TOTAL ENTROPY}

The conservation of the total energy-momentum tensor and the evolution of the chiral and vector currents determine the relativistic form of the second law of thermodynamics. If the four-divergence of the entropy four-vector is to be positive semidefinite (as implied by the generalized second law) the chiral and vector currents must contain supplementary kinetic coefficients corresponding to the hypermagnetic and to the vortical currents. In what follows the dissipative effects are included in the framework of the Landau approach: Tthe total four-velocity coincides then with the velocity of the energy transport defined from the mixed components of the total energy-momentum tensor.

\section{A. Ohmic and chiral currents}

In the simplest situation the total energy-momentum tensor of the system $\left(T_{\mu \nu}^{(\mathrm{tot})}\right)$ consists of four qualitatively different contributions: the energy-momentum tensor of the charged species (denoted by $T_{\mu \nu}^{( \pm)}$), the energy-momentum tensor of the chiral species (labeled by $T_{\mu \nu}^{(R)}$ ), the dissipative contribution $\left(T_{\mu \nu}^{(\text {diss })}\right)$ and the gauge contribution $\left(\mathcal{T}_{\mu \nu}\right)$ (corresponding to an hypercharge gauge field strength $Y_{\mu \nu}$ ):

$$
T_{\mu \nu}^{(\mathrm{tot})}=T_{\mu \nu}^{(+)}+T_{\mu \nu}^{(-)}+T_{\mu \nu}^{(R)}+T_{\mu \nu}^{(\mathrm{diss})}+\mathcal{T}_{\mu \nu} .
$$

The covariant conservation of $T_{\mu \nu}^{(\mathrm{tot})}$ implies, as usual, that $\nabla_{\mu} T_{\text {(tot) }}^{\mu \nu}=0$ where $\nabla_{\mu}$ denotes the covariant derivative ${ }^{1}$ defined from the metric tensor $g_{\mu \nu}$ (with signature mostly minus). The chiral and the conduction currents coexist but are not bound to coincide: They obey different equations. More specifically the anomalous current is not covariantly conserved, and its evolution can be written as ${ }^{2}$

$$
\nabla_{\mu} j_{R}^{\mu}=\mathcal{A}_{R} Y_{\alpha \beta} \tilde{Y}^{\alpha \beta}, \quad j_{R}^{\mu}=\tilde{n}_{R} u_{R}^{\mu}+\nu_{R}^{\mu},
$$

where $\mathcal{A}_{R}$ is a numerical factor that is determined by the specific nature of the chiral species and by the coupling to the hypercharge field; note that in the Landau frame $\nu_{R}^{\mu} u_{\mu}^{R}=0$. Conversely the conduction current is covariantly

\footnotetext{
${ }^{1}$ The discussion is conducted in a general relativistic formulation even if the spectrum of AMHD is discussed in flat space-time.

${ }^{2}$ Note that $\tilde{n}_{R}$ and $u_{R}^{\mu}$ are, respectively, the concentration and the four-velocity of the chiral species.
}

conserved. It is a source of the evolution equations of the gauge fields, and it may even contain a dissipative contribution:

$$
\nabla_{\mu} Y^{\mu \nu}=4 \pi j^{\nu}, \quad j^{\nu}=j_{+}^{\nu}+j_{-}^{\nu}, \quad \nabla_{\mu} j^{\mu}=0,
$$

where $j_{ \pm}^{\nu}=\left(q_{ \pm} \tilde{n}_{ \pm} u_{ \pm}^{\mu}+\nu_{ \pm}^{\mu}\right)$. The dual field strength $\tilde{Y}^{\mu \nu}$ obeys, as usual, $\nabla_{\mu} \tilde{Y}^{\mu \nu}=0$. This is the approach followed in [7] which differs from other more conventional approaches (see, for instance, Ref. [15], and especially Ref. [16]) where the anomalous current and the conduction current are identified.

In the present approach the anomalous current is not directly the source of the evolution of the hypercharge. Indeed by looking at Eq. (2.3) it is clear that $j^{\mu}$ is covariantly conserved, but it would be anomalous in the discussion of $[15,16]$. When the conserved current is Ohmic the entropy will also increase thanks to the Joule heating. The kinetic coefficients will always be determined from the requirement that the four-divergence of the entropy four-vector is positive semidefinite. In summary the present investigation deals with the situation where anomalous currents are present together with conserved currents. While this system, firstly analyzed in [7], differs from the one of Ref. [16] we always use the relativistic generalization of the second principle of thermodynamics to determine, at least partially, the kinetic coefficients.

In a general relativistic description $Y_{\mu \nu}$ and its dual account for the evolution of the hypercharge field ${ }^{3}$; however, the gauge field strength can be decomposed into the hyperelectric and hypermagnetic parts denoted, respectively, by $\mathcal{E}^{\mu}$ and $\mathcal{B}^{\mu}$ :

$$
Y_{\alpha \beta}=\mathcal{E}_{\alpha} u_{\beta}-\mathcal{E}_{\beta} u_{\alpha}+E_{\alpha \beta \rho \sigma} u^{\rho} \mathcal{B}^{\sigma},
$$

where $E_{\alpha \beta \rho \sigma}=\sqrt{-g} \epsilon_{\alpha \beta \rho \sigma}$ and $\epsilon_{\alpha \beta \rho \sigma}$ is the four-dimensional Levi-Civita symbol while $g$ is the determinant of the metric tensor. The total four-velocity of the system follows from

$$
(p+\rho) u^{\mu} u^{\nu}=\sum_{a}\left[p_{(a)}+\rho_{(a)}\right] u_{(a)}^{\mu} u_{(a)}^{\nu},
$$

where $w=(\rho+p)$ denotes the total enthalpy density; the sum in Eq. (2.5) runs over all the species of the plasma, both charged and chiral. Close to an equilibrium situation the four-velocity of the anomalous species coincides with the bulk velocity of the plasma and, therefore, $u_{R}^{\mu} \simeq u^{\mu}$. The vorticity four-vector can then be defined as

\footnotetext{
${ }^{3}$ As soon as we speak of hyperelectric and hypermagnetic fields we are implicitly assuming that the plasma has a finite conduction current so that a preferred frame can be selected where the electric fields are suppressed. Even if the electric and magnetic fields are nonrelativistic concepts, it is practical to introduce the electric and the magnetic components of the gauge field strength in a generally covariant language.
} 


$$
\omega^{\mu}=\tilde{f}^{\mu \alpha} u_{\alpha} \equiv \frac{1}{2} E^{\mu \alpha \beta \gamma} u_{\alpha} f_{\beta \gamma}, \quad f_{\beta \gamma}=\nabla_{\beta} u_{\gamma}-\nabla_{\gamma} u_{\beta} .
$$

From Eqs. (2.4) and (2.6) it follows that the fourdivergences of $\mathcal{E}^{\mu}, \mathcal{B}^{\mu}$ and $\omega^{\mu}$ are given by

$$
\begin{gathered}
w \nabla_{\mu} \omega^{\mu}=-2 \omega^{\alpha} \partial_{\alpha} p-2 \tilde{n} \mathcal{E}^{\alpha} \omega_{\alpha} \\
w \nabla_{\mu} \mathcal{B}^{\mu}=2 w Y_{\rho \sigma} \omega^{\rho} u^{\sigma}+u_{\mu} \partial_{\alpha} p \tilde{Y}^{\mu \alpha}+u_{\mu} Y_{\alpha \beta} j^{\beta} \tilde{Y}^{\mu \alpha} \\
w \nabla_{\mu} \mathcal{E}^{\mu}=w\left[4 \pi j^{\alpha} u_{\alpha}-\tilde{Y}^{\mu \rho} \omega_{\mu} u_{\rho}\right]+Y^{\beta \gamma} u_{\beta} \partial_{\gamma} p+Y^{\beta \gamma} u_{\beta} Y_{\gamma \alpha} j^{\alpha} .
\end{gathered}
$$

Equations (2.7), (2.8) and (2.9) have been obtained in the globally neutral case where $\tilde{n}=\tilde{n}_{+}=\tilde{n}_{-}$and $q_{+}=q=-q_{-}$, but they can be easily generalized to the case where the plasma is not globally neutral.

\section{B. First and second principles of thermodynamics}

Denoting with $\mu_{R}$ the chemical potential associated with the anomalous species, the first principle of thermodynamics demands

$$
\begin{gathered}
d E=T d S-p d V+\mu_{R} d N_{R}, \\
w=\rho+p=T \varsigma+\mu_{R} \tilde{n}_{R} .
\end{gathered}
$$

The fundamental identity $E=T S-p V+\mu_{R} N_{R}$ can be divided by a fiducial volume, and the result is the one reported in the second relation of Eq. (2.10) where $\varsigma$ is the entropy density and $\rho$ the total energy density of the system. Combining the two relations of Eq. (2.10) further thermodynamic relations can be obtained. ${ }^{4}$ Since the anomalyinduced currents are protected by topology they are not associated with dissipative effects. Thus, the entropy production of the plasma must only come, in the relativistic case, from the viscosity coefficients or from the Ohmic contributions but neither from the chiral currents nor from the corresponding diffusive contribution. The absence of dissipative contributions stemming from the anomalous sector demands that the total entropy four-vector must be supplemented by two further coefficients $\mathcal{S}_{\omega}$ and $\mathcal{S}_{B}$ :

$$
\varsigma^{\mu}=\varsigma u^{\mu}-\bar{\mu}_{R} \nu_{R}^{\mu}+\mathcal{S}_{\omega} \omega^{\mu}+\mathcal{S}_{B} \mathcal{B}^{\mu},
$$

where we introduced a rescaled chemical potential $\bar{\mu}_{R}=\mu_{R} / T$. The covariant conservation of the total energy momentum tensor $T_{\mu \nu}^{(\text {tot })}$ can be written as

$$
\nabla_{\mu} \varsigma^{\mu}-\frac{\sigma}{T} Y^{\alpha \beta} Y_{\nu \alpha} u^{\nu} u_{\beta}-\frac{T_{(\mathrm{diss})}^{\mu \nu}}{T} \nabla_{\mu} u_{\nu}=\mathcal{Z}
$$

${ }^{4}$ Like, for instance, $\varsigma \partial_{\alpha} T+\tilde{n}_{R} \partial_{\alpha} \mu_{R}=\partial_{\alpha} p$ or $\partial_{\alpha} \rho=T \partial_{\alpha} \varsigma+$ $\mu_{R} \partial_{\alpha} \tilde{n}_{R}$. where we assumed, for the sake of simplicity, a global charge neutrality of the plasma and a corresponding Ohmic form for the charged species, namely $\mathcal{P}_{\mu}^{\alpha} j^{\mu}=\sigma Y^{\alpha \nu} u_{\nu}$ where $\mathcal{P}_{\mu}^{\alpha}=\delta_{\mu}^{\alpha}-u_{\mu} u^{\alpha}$ is the standard projector. The function $\mathcal{Z}$ appearing in Eq. (2.12) is given by

$$
\begin{aligned}
\mathcal{Z}= & \nabla_{\mu}\left(\mathcal{S}_{\omega} \omega^{\mu}+\mathcal{S}_{B} \mathcal{B}^{\mu}\right)-\frac{\nu^{\alpha} u^{\beta}}{T} Y_{\alpha \beta}-\partial_{\beta} \bar{\mu}_{R} \nu_{R}^{\beta} \\
& -\mathcal{A}_{R} \bar{\mu}_{R} Y_{\alpha \beta} \tilde{Y}^{\alpha \beta} .
\end{aligned}
$$

We remark that the specific definition of the entropy fourvector depends on the chemical potential of the system. However, since the coefficient $\mathcal{A}_{R}$ does not have a definite sign, the anomalous currents may even lead to violation of the second principle of thermodynamics unless $\mathcal{Z}$ vanishes identically.

\section{Magnetic and vortical coefficients}

The vortical and the magnetic currents modify also the diffusive contributions denoted, respectively, by $\nu^{\alpha}$ and $\nu_{R}^{\alpha}$ in Eq. (2.13). Four different coefficients parametrize the relation between $\left(\nu^{\alpha}, \nu_{R}^{\alpha}\right)$ and $\left(\omega^{\alpha}, \mathcal{B}^{\alpha}\right)$ :

$$
\nu^{\alpha}=\Lambda_{\omega} \omega^{\alpha}+\Lambda_{B} \mathcal{B}^{\alpha}, \quad \nu_{R}^{\alpha}=\Lambda_{R \omega} \omega^{\alpha}+\Lambda_{R B} \mathcal{B}^{\alpha},
$$

where $\left(\Lambda_{\omega}, \Lambda_{B}\right)$ and $\left(\Lambda_{R \omega}, \Lambda_{R B}\right)$ all depend on the chemical potential and on the temperature. Using Eqs. (2.7), (2.8) and (2.9) the condition $\mathcal{Z}=0$ together with the explicit expression of $\mathcal{Z}$ [see Eq. (2.13)] becomes

$$
\begin{gathered}
{\left[2 \mathcal{S}_{B}-\left(\frac{\Lambda_{\omega}}{T}\right)\right]\left(\omega^{\alpha} \mathcal{B}_{\alpha}\right)+\left[4 \bar{\mu}_{R} \mathcal{A}_{R}-\left(\frac{\Lambda_{B}}{T}\right)\right]\left(\mathcal{E}^{\alpha} \mathcal{B}_{\alpha}\right)} \\
-\frac{2}{w} \sigma_{\mathrm{c}} \omega^{\alpha} \mathcal{E}^{\beta} u^{\mu} \mathcal{B}^{\nu} E_{\alpha \beta \mu \nu} \mathcal{S}_{\omega}+\omega^{\alpha} \mathcal{P}_{\alpha}+\mathcal{B}^{\alpha} \mathcal{Q}_{\alpha}=0
\end{gathered}
$$

where $\mathcal{P}_{\alpha}$ and $\mathcal{Q}_{\alpha}$ are two differential operators defined respectively, as

$$
\begin{aligned}
& \mathcal{P}_{\alpha}=\partial_{\alpha} \mathcal{S}_{\omega}-\frac{2}{w} \mathcal{S}_{\omega} \partial_{\alpha} p-\partial_{\alpha} \bar{\mu}_{R} \Lambda_{R \omega}, \\
& \mathcal{Q}_{\alpha}=\partial_{\alpha} \mathcal{S}_{B}-\frac{\mathcal{S}_{B}}{w} \mathcal{S}_{\omega} \partial_{\alpha} p-\partial_{\alpha} \bar{\mu}_{R} \Lambda_{R B}
\end{aligned}
$$

The results of Eqs. (2.13)-(2.15) follow easily if we recall that, by definition, $u^{\alpha} \omega_{\alpha}, u^{\beta} \mathcal{E}_{\beta}$ and $u^{\gamma} \mathcal{B}_{\gamma}$ are all vanishing.

To satisfy the condition expressed by Eq. (2.15) the fourvectors multiplying $\omega^{\alpha}$ and $\mathcal{B}^{\alpha}$ must vanish together with the coefficients of the terms multiplied by $\omega^{\alpha} \mathcal{B}_{\alpha}$ and $\mathcal{E}^{\alpha} \mathcal{B}_{\alpha}$. We then arrive at the following conditions:

$\mathcal{P}_{\alpha}=0, \quad \mathcal{Q}_{\alpha}=0, \quad \Lambda_{B}=4 \mu_{R} \mathcal{A}_{R}$,

$\Lambda_{\omega}=2 T \mathcal{S}_{B}, \quad \mathcal{S}_{\omega}=0$.

If, as established, $\mathcal{S}_{\omega}=0$ then Eq. (2.15) also implies that $\Lambda_{R \omega}=0$. All the coefficients we ought to determine 
depend on $\bar{\mu}_{R}$ and on the pressure. Thus, the conditions of Eq. (2.17) are equivalent to the following system of equations:

$$
\left(\frac{\partial \mathcal{S}_{B}}{\partial p}-\frac{\mathcal{S}_{B}}{w}\right) \partial_{\alpha} p+\left(\frac{\partial \mathcal{S}_{B}}{\partial \bar{\mu}_{R}}-\Lambda_{R B}\right) \partial_{\alpha} \bar{\mu}_{R}=0,
$$

where $\Lambda_{\omega}=2 T \mathcal{S}_{B}$ and $\Lambda_{B}=4 \mathcal{A}_{R} \bar{\mu}_{R} T$. Using some standard thermodynamic relations (giving the partial derivatives of the pressure and of the rescaled chemical potential with respect to the temperature) the various kinetic coefficients can be determined, after some algebra:

$$
\mathcal{S}_{B}\left(\bar{\mu}_{R}, T\right)=T a_{B}\left(\bar{\mu}_{R}\right), \quad \Lambda_{R B}=\frac{\partial}{\partial \bar{\mu}_{R}}\left[T a_{B}\left(\bar{\mu}_{R}\right)\right],
$$

$\Lambda_{\omega}\left(\bar{\mu}_{R}, T\right)=2 T^{2} a_{B}\left(\bar{\mu}_{R}\right), \quad \Lambda_{B}\left(\bar{\mu}_{R}, T\right)=4 \mathcal{A}_{R} \bar{\mu}_{R} T$,

where $a_{B}\left(\bar{\mu}_{R}\right)$ is an arbitrary function of the rescaled chemical potential. Note also that $\Lambda_{B}$ is fully determined in terms of the coefficient of the anomaly, and it is, in practice, only a function of the chemical potential itself since, by definition, $\bar{\mu}_{R} T=\mu_{R}$.

In summary, in a globally neutral plasma with an anomalous current, the relativistic second law implies that the nonanomalous current must contain magnetic and vortical contributions. If the plasma is not hypercharge neutral the form of the kinetic coefficients is subjected to a higher degree of arbitrariness since a second chemical potential must be introduced in the analysis (see the appendix of Ref. [7]).

Even if this last issue has been thoroughly discussed in [7], it is nonetheless useful to add further explanatory details. For this purpose, let us consider, for instance, the fundamental identity of thermodynamics already written in the second relation reported in Eq. (2.10). If the plasma is not neutral Eq. (2.10) gets modified as $w=T \varsigma+\mu_{R} \tilde{n}_{R}+$ $\mu_{+} \tilde{n}_{+}+\mu_{-} \tilde{n}_{-}$where $\tilde{n}_{ \pm}$are the concentrations of the positively and negatively charged species. Recalling that $\mu_{+}=-\mu_{-}=\mu$ we have that the enthalpy density can be easily written as $w=T \varsigma+\mu_{R} \tilde{n}_{R}+\mu \tilde{n}$ where $\tilde{n}=\left(\tilde{n}_{+}-\tilde{n}_{-}\right)$. It is clear that in the globally neutral case (i.e. when the positive charges are balanced by the negative charges) the obtained equation exactly reproduces Eq. (2.10).

If the plasma is not globally neutral the different expression of the enthalpy density induces differences in all the other equations. For instance, the first differential relation mentioned in the footnote after Eq. (2.10) is modified as $\varsigma \partial_{\alpha} T+\tilde{n}_{R} \partial_{\alpha} \mu_{R}+\tilde{n} \partial_{\alpha} \mu=\partial_{\alpha} p$. Going through all the different steps of the derivation (see the appendix of [7]) it is possible to appreciate that the kinetic coefficients are underdetermined, and as a consequence they suffer a higher degree of arbitrariness.

\section{HIGH-FREQUENCY PROPAGATION}

\section{A. Two-fluid AMHD equations}

The purpose of this section is to establish a direct connection between the spectrum of anomalous plasmas and the spectrum of conventional (cold) plasmas. ${ }^{5}$ For this purpose we preferentially consider the situation where the charged species are massive. Furthermore, as already mentioned, the plasma is taken as globally neutral, i.e. characterized by a common concentration of positive and negative charge carriers. The energy densities of the charged species are denoted by $\tilde{\rho}_{ \pm}$. In terms of the concentrations $\tilde{n}_{ \pm}$and of the masses $m_{ \pm}$they are also given by $\tilde{\rho}_{ \pm}=\tilde{n}_{ \pm}\left(m_{ \pm}+3 T_{ \pm} / 2\right)$ where $T_{ \pm}$are the temperatures of the charged species. In approximate thermal equilibrium $T_{+} \simeq T_{-} \sim T$. Moreover, in the case of a cold plasma $T_{ \pm} / m_{ \pm} \ll 1$. Since $p_{ \pm}=\tilde{n}_{ \pm} T_{ \pm}$we also have that $p_{ \pm} \ll \tilde{\rho}_{ \pm}$.

From Eq. (2.14) the dissipative contributions to the current densities are

$$
\begin{aligned}
\vec{j} & =q\left(\tilde{n}_{+} \vec{v}_{+}-\tilde{n}_{-} \vec{v}_{-}\right)+\Lambda_{\omega} \vec{\omega}-\Lambda_{B} \vec{B}, \\
\vec{j}_{R} & =\tilde{n}_{R} \vec{v}_{R}+\Lambda_{R \omega} \vec{\omega}-\Lambda_{R B} \vec{B},
\end{aligned}
$$

where $\vec{B}$ is the hypermagnetic field and $\vec{\omega}$ is the vorticity three-vector. Both quantities can be deduced as the spatial components of $\mathcal{B}^{\mu}$ and $\omega^{\mu}$. The three-vector where $\vec{\omega}$ defines the total vorticity is $\vec{\omega}=\left(\tilde{\rho}_{+} \vec{\omega}_{+}+\tilde{\rho}_{-} \vec{\omega}_{-}\right) /\left(\tilde{\rho}_{+}+\tilde{\rho}_{-}\right)$ and should not be confused with the frequency (denoted by $\Omega$ ). Furthermore, the hyperelectric fields are denoted by $\vec{E}$. The equations obeyed by $\vec{E}$ and $\vec{B}$ are

$$
\vec{\nabla} \cdot \vec{E}=4 \pi q\left(\tilde{n}_{+}-\tilde{n}_{-}\right), \quad \vec{\nabla} \times \vec{E}=-\partial_{t} \vec{B},
$$

$\vec{\nabla} \times \vec{B}=4 \pi q\left(\tilde{n}_{+} \vec{v}_{+}-\tilde{n}_{-} \vec{v}_{-}\right)+c_{\omega} \vec{\omega}-c_{B} \vec{B}+\partial_{t} \vec{E}$,

where $\vec{B}$ is divergenceless (i.e. $\vec{\nabla} \cdot \vec{B}=0$ ). Note that $c_{\omega}=$ $4 \pi \Lambda_{\omega}$ and $c_{B}=4 \pi \Lambda_{B}$. Since the divergence of the entropy four-vector must be positive semidefinite Eqs. (2.19) and (2.20) determine $\Lambda_{\omega}$ and $\Lambda_{B}$. Instead of carrying various numerical factors it is more convenient to define the rescaled kinetic coefficients $c_{\omega}$ and $c_{B}$ which directly enter Eq. (3.3):

$$
c_{\omega}\left(\bar{\mu}_{R}, T\right)=8 \pi T^{2} a_{B}\left(\bar{\mu}_{R}\right), \quad c_{B}\left(\bar{\mu}_{R}, T\right)=16 \pi \mathcal{A}_{R} \bar{\mu}_{R} T ;
$$

since $c_{\omega}$ and $c_{B}$ multiply the vortical and the magnetic terms they are referred to as the vortical and the magnetic coefficients. With a similar logic Eqs. (2.19) and (2.20) also fix $\Lambda_{R \omega}$ and $\Lambda_{R B}$. We can therefore define two further coefficients $c_{R \omega}$ and $c_{R B}$ :

\footnotetext{
${ }^{5}$ Cold plasmas are rather common systems ranging from the ionosphere to some regions of the interstellar medium (see e.g. [1-3]).
} 


$$
c_{R B}\left(\bar{\mu}_{R}, T\right)=\frac{\partial}{\partial \bar{\mu}_{R}}\left[T a_{B}\left(\bar{\mu}_{R}\right)\right], \quad c_{R \omega}\left(\bar{\mu}_{R}, T\right)=0,
$$

affecting directly the evolution of the concentrations

$$
\begin{gathered}
\partial_{t} \tilde{n}_{+}+\vec{\nabla} \cdot\left(\tilde{n}_{+} \vec{v}_{+}\right)+\frac{1}{q} \vec{\nabla} \cdot\left(c_{\omega} \lambda_{+} \vec{\omega}_{+}\right) \\
-\frac{1}{q} \vec{\nabla} \cdot\left(c_{B} \lambda_{+} \vec{B}\right)=0, \\
\partial_{t} \tilde{n}_{-}+\vec{\nabla} \cdot\left(\tilde{n}_{-} \vec{v}_{-}\right)-\frac{1}{q} \vec{\nabla} \cdot\left(c_{\omega} \lambda_{-} \vec{\omega}_{-}\right) \\
+\frac{1}{q} \vec{\nabla} \cdot\left(c_{B} \lambda_{-} \vec{B}\right)=0, \\
\partial_{t} \tilde{n}_{R}+\vec{\nabla} \cdot\left(\tilde{n}_{R} \vec{v}_{R}\right)+\vec{\nabla} \cdot\left(c_{R \omega} \vec{\omega}\right)-\vec{\nabla} \cdot\left(c_{R B} \vec{B}\right) \\
=-4 \mathcal{A}_{\mathcal{R}} \vec{E} \cdot \vec{B}
\end{gathered}
$$

where $\lambda_{ \pm}=\tilde{\rho}_{ \pm} /\left(\tilde{\rho}_{+}+\tilde{\rho}_{-}\right)$. Concerning Eqs. (3.2) and (3.3) and Eqs. (3.6)-(3.8) few comments are in order. If $\tilde{n}_{+} \neq \tilde{n}_{-}$a second chemical potential $\mu_{Y}$ (corresponding to the hypercharge) can be introduced in Eq. (2.10). The global hypercharge neutrality of the plasma implies $\mu_{Y}=0$. The peculiar velocities determining the currents obey the following set of equations:

$$
\begin{gathered}
\partial_{t} \vec{v}_{-}+\left(\vec{v}_{-} \cdot \vec{\nabla}\right) \vec{v}_{-} \\
=-q \frac{\tilde{n}_{-}}{\tilde{\rho}_{-}}\left[\vec{E}+\vec{v}_{-} \times \vec{B}\right]+\Gamma_{c}\left(\vec{v}_{+}-\vec{v}_{-}\right)-\frac{\vec{\nabla} p_{+}}{\rho_{+}}, \\
\partial_{t} \vec{v}_{+}+\left(\vec{v}_{+} \cdot \vec{\nabla}\right) \vec{v}_{+}=q \frac{\tilde{n}_{+}}{\tilde{\rho}_{+}}\left[\vec{E}+\vec{v}_{+} \times \vec{B}\right] \\
+\Gamma_{c} \frac{\tilde{\rho}_{-}}{\tilde{\rho}_{+}}\left(\vec{v}_{-}-\vec{v}_{+}\right)-\frac{\vec{\nabla} p_{-}}{\rho_{-}}, \\
\partial_{t} \vec{v}_{R}+\left(\vec{v}_{R} \cdot \vec{\nabla}\right) \vec{v}_{R}=0 .
\end{gathered}
$$

Concerning Eqs. (3.9), (3.10) and (3.11) two comments are in order: The pressure gradients are eventually neglected, and $\Gamma_{c}$ denotes the collision frequency (i.e. the typical velocity divided by the mean free path). In connection with the first point it is clear that the dielectric properties of the plasmas are altered by the addition of pressure gradients, and this is why the first step is always to consider a homogeneous plasma eventually supplemented by an external magnetic field. Note that the collision frequency determines the generalized conductivity in the single fluid limit.

\section{B. Linearization of the two-fluid equations}

Equations (3.2)-(3.3), (3.6)-(3.8) and (3.9)-(3.11) are now linearized in the presence of the weak background magnetic field $\vec{B}_{0}$ with the aim of deriving the dispersion relations. The background field is considered homogeneous: This means that the variation of $\vec{B}_{0}$ occurs over typical length scales ${ }^{6}$ much larger than $1 / c_{B}$. The fluctuations of the various quantities are introduced as follows:

$$
\begin{aligned}
\tilde{n}_{ \pm}(t, \vec{x}) & =n_{0}+\delta \tilde{n}_{ \pm}(t, \vec{x}), \quad \tilde{n}_{R}(t, \vec{x})=n_{1}+\delta \tilde{n}_{R}(t, \vec{x}) \\
\vec{B}(t, \vec{x}) & =\vec{B}_{0}+\delta \vec{B}(t, \vec{x}),
\end{aligned}
$$

while for the other quantities [i.e. $\vec{v}_{ \pm}(t, \vec{x})=\delta \vec{v}_{ \pm}(t, \vec{x})$, $\vec{v}_{R}(t, \vec{x})=\delta \vec{v}_{R}(t, \vec{x})$ and $\left.\vec{E}(t, \vec{x})=\delta \vec{E}(t, \vec{x})\right]$ the fluctuations coincide with the field itself. In Eq. (3.12) $n_{0}$ and $n_{1}$ are, respectively, the uniform background charge and the uniform chiral concentration. The homogeneous value of the chemical potential is related to $n_{1}$, and the kinetic coefficients will also be homogeneous. In the case of approximate thermal equilibrium the chemical potential can be related to the concentration as $\bar{\mu}_{R}=\mu_{0} \tilde{n}_{R} / \varsigma$ where $\varsigma$ denotes the entropy density at equilibrium and where $\mu_{0}$ is a numerical constant. Therefore, if $\tilde{n}_{R}$ is perturbed around a homogeneous background the kinetic coefficients will also be, in the first approximation, homogeneous. Thanks to Eq. (3.12) the perturbed version of the evolution of the concentrations can be written as

$$
\begin{aligned}
\delta \tilde{n}_{ \pm}^{\prime}+n_{0}\left(\vec{\nabla} \cdot \delta \vec{v}_{ \pm}\right) & =0, \\
\delta \tilde{n}_{R}^{\prime}+n_{1}\left(\vec{\nabla} \cdot \delta \vec{v}_{R}\right) & =-4 \mathcal{A}_{\mathcal{R}} \delta \vec{E} \cdot \vec{B}_{0},
\end{aligned}
$$

where the prime denotes a derivation with respect to the time coordinate $t$. Since the kinetic coefficients are homogeneous in the first approximation, their contribution disappears from Eq. (3.13). With the same notations Eqs. (3.9), (3.10) and (3.11) imply instead

$$
\delta \vec{v}_{ \pm}^{\prime}= \pm \frac{q}{m_{ \pm}}\left[\delta \vec{E}+\delta \vec{v}_{ \pm} \times \vec{B}_{0}\right], \quad \delta \vec{v}_{R}^{\prime}=0 .
$$

In Eq. (3.14) the collision frequency $\Gamma_{c}$ [already introduced in Eqs. (3.9) and (3.10)] will become relevant at lower frequencies, as we see later. Finally, after inserting Eq. (3.12) into Eqs. (3.2) and (3.3) we obtain

$$
\begin{aligned}
\vec{\nabla} \cdot \delta \vec{E}=4 \pi q\left(\delta \tilde{n}_{+}-\delta \tilde{n}_{-}\right), \quad \vec{\nabla} \cdot \delta \vec{B}=0, & \\
\vec{\nabla} \times \delta \vec{E}=- & \delta \vec{B}^{\prime}, \\
\vec{\nabla} \times \delta \vec{B}= & \delta \vec{E}^{\prime}+4 \pi q n_{0}\left(\delta \vec{v}_{+}-\delta \vec{v}_{-}\right)-c_{B} \delta \vec{B} \\
& +c_{\omega}\left[\lambda_{+} \vec{\nabla} \times \delta \vec{v}_{+}+\lambda_{-} \vec{\nabla} \times \delta \vec{v}_{-}\right] .
\end{aligned}
$$

\footnotetext{
${ }^{6}$ In Sec. IV we specifically also discuss the opposite limit where $\vec{B}_{0}$ varies appreciably over typical lengths $L<1 / c_{B}$, and we see that, in this case, the background solution belongs to the class of Beltrami fields.
} 
From Eqs. (3.14) the equations obeyed by $\delta \vec{\omega}_{ \pm}$can also be deduced, and they are $\delta \vec{\omega}_{ \pm}^{\prime}= \pm q\left[-\delta \vec{B}^{\prime}+\vec{\nabla} \times\left(\delta \vec{v}_{ \pm} \times \vec{B}_{0}\right)\right] /$ $m_{ \pm}$. Recalling the standard vector identities ${ }^{7}$ the equation for $\delta \vec{\omega}_{ \pm}$can also be expressed as

$$
\delta \vec{\omega}_{ \pm}^{\prime}= \pm \frac{q}{m_{ \pm}}\left[-\delta \vec{B}^{\prime}-\vec{B}_{0}\left(\vec{\nabla} \cdot \delta \vec{v}_{ \pm}\right)+\left(\vec{B}_{0} \cdot \vec{\nabla}\right) \delta \vec{v}_{ \pm}\right] .
$$

From Eqs. (3.14) and (3.16) the relevant dispersion relations and the associated refraction indices can be obtained by treating separately the motions parallel and perpendicular to the magnetic field direction.

\section{Appleton-Hartree determinant}

While in conventional plasmas the Appleton-Hartree dispersion relation has been extensively discussed in the literature [6,17], the AMHD equations linearized in the twofluid limit contain vortical and magnetic currents. The Laplace transform of Eq. (3.16) implies the following equation:

$$
\begin{aligned}
(\vec{\nabla} \times \delta \vec{B})_{\Omega}= & -i \Omega \varepsilon_{s}(\Omega) \cdot \delta \vec{E}_{\Omega}-c_{B} \delta \vec{B}_{\Omega}+i c_{\omega} \vec{\nabla} \\
& \times\left[\varepsilon_{v}(\Omega) \cdot \delta \vec{E}_{\Omega}\right],
\end{aligned}
$$

where $\Omega$ is the frequency (not to be confused with the total vorticity). In Eq. (3.18) $\varepsilon_{s}(\Omega)$ and $\varepsilon_{v}(\Omega)$ denote, respectively, the standard and the vortical components of the dielectric tensor. The explicit form of $\varepsilon_{s}(\Omega)$ and $\varepsilon_{v}(\Omega)$ can be found in Appendix A; taking then the curl of Eq. (3.15) and using Eq. (3.18) we obtain the following equation:

$$
\begin{aligned}
\vec{\nabla} \times\left(\vec{\nabla} \times \delta \vec{E}_{\Omega}\right)= & \Omega^{2} \varepsilon_{s}(\Omega) \cdot \delta \vec{E}_{\Omega}-c_{B} \vec{\nabla} \times \delta \vec{E}_{\Omega} \\
& -\Omega c_{\omega} \vec{\nabla} \times\left[\varepsilon_{v}(\Omega) \cdot \delta \vec{E}_{\Omega}\right]
\end{aligned}
$$

We can now go to Fourier space and write Eq. (3.19) as

$$
\begin{aligned}
-\vec{k} \times \vec{k} \times \delta \vec{E}_{\vec{k} \Omega}= & \Omega^{2} \varepsilon_{s}(\Omega) \cdot \delta \vec{E}_{\vec{k} \Omega}-i c_{B} \vec{k} \times \delta \vec{E}_{\vec{k} \Omega} \\
& -i c_{\omega} \Omega \vec{k} \times\left[\varepsilon_{v}(\Omega) \cdot \delta \vec{E}_{\vec{k} \Omega}\right] .
\end{aligned}
$$

We can therefore introduce the refractive index ${ }^{8} n$ satisfying $n=k / \Omega$ where $k=|\vec{k}|$; choosing the coordinate system as $\vec{k}=(0, n \Omega \sin \theta, n \Omega \cos \theta)$ we can obtain from Eq. (3.20) the following Appleton-Hartree matrix:

$$
\left(\begin{array}{ccc}
{\left[1-\frac{\varepsilon_{1}}{n^{2}}+c_{\omega} \frac{\varepsilon_{4}}{n} c(\theta)\right]} & -i\left[\frac{\varepsilon_{2}}{n^{2}}+\frac{c_{B}}{n \Omega} c(\theta)+\frac{c_{\omega}}{n} \varepsilon_{3} c(\theta)\right] & i \frac{c_{B}}{n \Omega} s(\theta) \\
i\left[\frac{\varepsilon_{2}}{n^{2}}+\frac{c_{B}}{n \omega} c(\theta)+\frac{c_{\omega}}{n} \varepsilon_{3} c(\theta)\right] & {\left[c^{2}(\theta)-\frac{\varepsilon_{1}}{n^{2}}+\frac{\varepsilon_{4}(\Omega)}{n} c_{\omega} c(\theta)\right]} & -s(\theta) c(\theta) \\
-i \frac{c_{B}}{n \Omega} s(\theta)-i \frac{c_{\omega}}{n} \varepsilon_{3}(\Omega) & -s(\theta) c(\theta)+\frac{c_{\omega}}{n} \varepsilon_{4} s(\theta) & {\left[s^{2}(\theta)-\frac{\varepsilon_{\|}(\omega)}{n^{2}}\right]}
\end{array}\right) .
$$

The above matrix reduces to the standard form of the Appleton-Hartree matrix in the limit $c_{\omega} \rightarrow 0$ and $c_{B} \rightarrow 0$.

\section{DISPERSION RELATIONS}

The determinant of the Appleton-Hartree matrix obtained in Eq. (3.21) leads to the following expression:

$$
\begin{gathered}
\sin ^{2} \theta\left(\varepsilon_{\|}-n^{2}\right)\left[n^{2}\left(\varepsilon_{L}+\varepsilon_{R}\right)-2 \varepsilon_{R} \varepsilon_{L}\right]-2 \cos ^{2} \theta \varepsilon_{\|}\left(n^{2}-\varepsilon_{L}\right)\left(n^{2}-\varepsilon_{R}\right)+2 n^{6}\left[c_{B}^{2} f_{B}(\varepsilon, \Omega, n, \theta)+c_{B} g_{B}(\varepsilon, \Omega, n, \theta)\right. \\
\left.+c_{\omega}^{2} f_{\omega}(\varepsilon, \Omega, n, \theta)+c_{\omega} g_{\omega}(\varepsilon, \Omega, n, \theta) c_{B}^{2} c_{\omega} h_{1}(\varepsilon, \Omega, n, \theta)+c_{B} c_{\omega} h_{2}(\varepsilon, \Omega, n, \theta)+c_{\omega}^{2} c_{B} h_{3}(\varepsilon, \Omega, n, \theta)\right]=0 .
\end{gathered}
$$

Equation (4.1) is written in terms of the seven functions explicitly reported in Eq. (A7) of Appendix A. These functions have a specific dependence upon the dielectric tensors; with a collective notation such a dependence has been indicated by $\varepsilon$. The notations followed in Eq. (4.1) imply that $c_{B}^{2}$ multiplies $f_{B} ; c_{\omega}^{2}$ multiplies $f_{\omega} ; g_{B}$ and $g_{\omega}$ multiply, respectively, $c_{B}$ and $c_{\omega}$; the three functions $h_{1}, h_{2}$ and $h_{3}$ multiply instead the mixed products. Finally both in Eqs. (4.1) and in Eq. (A7) we have introduced $\varepsilon_{L}=\left(\varepsilon_{1}+\varepsilon_{2}\right)$ and $\varepsilon_{R}=\left(\varepsilon_{1}-\varepsilon_{2}\right)$ given by ${ }^{9}$

\footnotetext{
${ }^{7}$ In particular, we recall that $\vec{\nabla} \times(\vec{a} \times \vec{b})=[\vec{a}(\vec{\nabla} \cdot \vec{b})-\vec{b}(\vec{\nabla} \cdot \vec{a})+(\vec{b} \cdot \vec{\nabla}) \vec{a}-(\vec{a} \cdot \vec{\nabla}) \vec{b}]$.

${ }^{8}$ The refractive index cannot be confused with the concentrations denoted by $\tilde{n}_{ \pm}$and $\tilde{n}_{R}$; their homogeneous values $n_{0}$ and $n_{1}$ carry specific subscripts so that the notations are clearly established.

${ }^{9}$ The divergence of $\varepsilon_{L}$ (for $\Omega \rightarrow \Omega_{B+}$ ) and of $\varepsilon_{R}$ (for $\Omega \rightarrow \Omega_{B-}$ ) are nothing but the cyclotron resonances occurring in the case of conventional plasmas. If the mass of the ions is larger than the mass of the negatively charged species the cyclotron resonance for the ions occurs typically at a frequency which is lower in comparison with the cyclotron resonance of the electrons. The chiral magnetic and the chiral vortical terms appear in Eq. (4.1) and determine the dispersion relations. In spite of this they do not affect $\varepsilon_{L}$ nor $\varepsilon_{R}$.
} 


$$
\begin{aligned}
& \varepsilon_{L}(\Omega)=1-\frac{\Omega_{p}^{2}}{\left(\Omega+\Omega_{B-}\right)\left(\Omega-\Omega_{B+}\right)}, \\
& \varepsilon_{R}(\Omega)=1-\frac{\Omega_{p}^{2}}{\left(\Omega+\Omega_{B+}\right)\left(\Omega-\Omega_{B-}\right)},
\end{aligned}
$$

where $\Omega_{p}^{2}=\left(\Omega_{p+}^{2}+\Omega_{p-}^{2}\right)$. When $c_{B}=c_{\omega}=0$ the magnetic and the vortical currents are absent from the twofluid AMHD equations, and Eq. (4.1) implies the standard result [6]:

$$
\begin{gathered}
\sin ^{2} \theta\left(\frac{1}{n^{2}}-\frac{1}{\varepsilon_{\|}}\right)\left[\frac{1}{2}\left(\frac{1}{\varepsilon_{L}}+\frac{1}{\varepsilon_{R}}\right)-\frac{1}{n^{2}}\right] \\
=\cos ^{2} \theta\left(\frac{1}{\varepsilon_{L}}-\frac{1}{n^{2}}\right)\left(\frac{1}{\varepsilon_{R}}-\frac{1}{n^{2}}\right) .
\end{gathered}
$$

The dispersion relations for a wave propagating parallel (i.e. $\theta=0$ ) and perpendicular (i.e. $\theta=\pi / 2$ ) to the magnetic field direction can be easily derived from Eq. (4.3). If $\theta=0$ Eq. (4.3) reduces to $\left(n^{2}-\varepsilon_{\mathrm{R}}\right)\left(n^{2}-\varepsilon_{\mathrm{L}}\right)=0$ while for $\theta=\pi / 2$ Eq. (4.3) implies $\left(n^{2}-\varepsilon_{\|}\right)\left[n^{2}\left(\varepsilon_{\mathrm{L}}+\varepsilon_{\mathrm{R}}\right)-\right.$ $\left.2 \varepsilon_{\mathrm{L}} \varepsilon_{\mathrm{R}}\right]=0$. These dispersion relations give therefore the conventional results ${ }^{10}$ which are generalized hereunder.

\section{A. Free-field propagation}

In the absence of a magnetic field there is no preferred direction, and the dispersion relations follow from Eqs. (4.3) and (A7) by setting all the Larmor frequencies to zero. In this case $\varepsilon_{R}=\varepsilon_{L}=\varepsilon_{\|}$, and the dispersion relations stem from the following two conditions, namely

$$
\varepsilon_{\|}(\Omega)=0, \quad\left(n^{2}-\varepsilon_{\|}\right) \Omega \mp n c_{B}=0 .
$$

Equation (4.4) demonstrates that the vortical current does not contribute to the dispersion relations in the free-field case: $c_{\omega}$ is absent from Eq. (4.4) since the two-fluid effects cancel in the total vorticity. This cancellation is either exact (as in the case of the free-field propagation) or approximate (as we see later in the presence of the magnetic field). Indeed, as it can be explicitly verified from Eqs. (A1), (A5) and (A6), $\epsilon_{v}(\Omega) \rightarrow 0$ when $B_{0} \rightarrow 0$ : In the limit $B_{0} \rightarrow 0$ the vorticity of positively and negatively charged species is balanced so that the net total vorticity vanishes.

The dispersion relation $\varepsilon_{\|}(\Omega)=0$ implies $\Omega=\Omega_{p}$. This wave does not propagate since its group velocity vanishes, and these are nothing but the electrostatic plasma

\footnotetext{
${ }^{10}$ Along $\theta=0$ we thus obtain usual dispersion relations for the two circular polarizations of the electromagnetic wave, i.e. $n^{2}=$ $\varepsilon_{\mathrm{R}}$ and $n^{2}=\varepsilon_{\mathrm{L}}$, while along $\theta=\pi / 2$ we have the dispersion relations for the "ordinary" (i.e. $n^{2}=\varepsilon_{\|}$) and "extraordinary" [i.e. $\left.n^{2}=2 \varepsilon_{\mathrm{R}} \varepsilon_{\mathrm{L}} /\left(\varepsilon_{\mathrm{R}}+\varepsilon_{\mathrm{L}}\right)\right]$ plasma waves.
}

oscillations [6]. The solution of the second equation in Eq. (4.4) is instead ${ }^{11}$

$$
n= \pm \frac{c_{B}}{2 \Omega}+\sqrt{1-\frac{\Omega_{p}^{2}}{\Omega^{2}}+\frac{c_{B}^{2}}{4 \Omega^{2}}} .
$$

Equation (4.5) implies also $\Omega^{2}=\Omega_{p}^{2}+k^{2} \mp k c_{B}$; these modes are propagating but only affected by the magnetic current, as previously remarked. The birefringent nature of the dispersion relations are discussed a bit later since this free-field effect may interfere with the presence of the background magnetic field.

If $c_{B} \rightarrow 0$ and $c_{\omega} \rightarrow 0$ we have that $n \rightarrow 0$ whenever one of the following three possibility are separately verified $\varepsilon_{\|}(\Omega)=0$ or $\varepsilon_{L}(\Omega)=0$ or even $\varepsilon_{R}(\Omega)=0$. The frequencies arising from the previous conditions are cutoffs because, for given equilibrium conditions, they define frequencies above or below which the wave ceases to propagate at any angle $\left(k \rightarrow 0\right.$ for finite $\Omega$, i.e. $v_{p}=$ $\Omega / k \rightarrow \infty$ and $v_{g} \rightarrow 0$ where $v_{p}$ and $v_{g}$ are, respectively, the phase and the group velocities). This is what happens, in particular, with the dispersion relation of Eq. (4.5). Let us finally remark that the remaining two cutoffs stemming from the conditions $\varepsilon_{L}(\Omega)=0$ and $\varepsilon_{R}(\Omega)=0$ in Eqs. (4.2) are given, respectively, by

$\Omega_{R}=\sqrt{\Omega_{p}^{2}+\left(\Omega_{B+}+\Omega_{B-}\right)^{2} / 4}-\left(\Omega_{B+}+\Omega_{B-}\right) / 2$,

$\Omega_{L}=\sqrt{\Omega_{p}^{2}+\left(\Omega_{B+}+\Omega_{B-}\right)^{2} / 4}+\left(\Omega_{B+}+\Omega_{B-}\right) / 2$.

\section{B. Parallel propagation}

Taking the limit $\theta \rightarrow 0$ in Eq. (4.1) and recalling the results of Eq. (A7) we obtain

$$
\begin{aligned}
& \varepsilon_{\|}\left\{n c_{B}+\left[n^{2}+n c_{\omega}\left(\varepsilon_{3}+\varepsilon_{4}\right)-\varepsilon_{R}\right] \Omega\right\} \\
& \quad \times\left\{n c_{B}-\left[n^{2}+n c_{\omega}\left(-\varepsilon_{3}+\varepsilon_{4}\right)-\varepsilon_{L}\right] \Omega\right\}=0 .
\end{aligned}
$$

If $\varepsilon_{\|}(\Omega)=0$ we go back to the case of electrostatic oscillations. Therefore, assuming $\varepsilon_{\|}(\Omega) \neq 0$, Eq. (4.8) implies that the standard dispersion relations are modified as

$$
\begin{aligned}
n= & \frac{1}{2 \Omega}\left[-c_{B}-c_{\omega}\left(\varepsilon_{3}+\varepsilon_{4}\right) \Omega\right. \\
& \left. \pm \sqrt{4 \varepsilon_{R} \Omega^{2}+\left[c_{B}+c_{\omega} \Omega\left(\varepsilon_{3}+\varepsilon_{4}\right)\right]^{2}}\right],
\end{aligned}
$$

\footnotetext{
${ }^{11}$ The positive square root has been chosen in Eq. (4.5) in order to get $\Omega>0$; we consider only positive $\Omega$ since solutions with $\Omega<0$ simply correspond to waves traveling in the opposite direction.
} 


$$
\begin{aligned}
n= & \frac{1}{2 \Omega}\left[c_{B}+c_{\omega}\left(\varepsilon_{3}-\varepsilon_{4}\right) \Omega\right. \\
& \left. \pm \sqrt{4 \varepsilon_{L} \Omega^{2}+\left[c_{B}+c_{\omega} \Omega\left(\varepsilon_{3}-\varepsilon_{4}\right)\right]^{2}}\right] .
\end{aligned}
$$

Thus the dispersion relations for the generalized $L$-mode and $R$-mode are given, respectively, by

$$
\begin{aligned}
& \Omega^{2} \varepsilon_{R}(\Omega)=k^{2}+k\left[c_{B}+c_{\omega}\left(\varepsilon_{3}+\varepsilon_{4}\right) \Omega\right], \\
& \Omega^{2} \varepsilon_{L}(\Omega)=k^{2}-k\left[c_{B}+c_{\omega}\left(\varepsilon_{3}-\varepsilon_{4}\right) \Omega\right] .
\end{aligned}
$$

In the high-frequency limit (i.e. formally ${ }^{12} \Omega \rightarrow \infty$ ) we have that $c_{\omega}\left(\varepsilon_{3} \pm \varepsilon_{4}\right) \Omega \rightarrow 0$ since, from Eqs. (A5) and (A6), we have

$$
\begin{aligned}
& c_{\omega}\left(\varepsilon_{3}+\varepsilon_{4}\right) \Omega=\frac{q c_{\omega}}{\left(m_{+}+m_{-}\right)}\left[\frac{\Omega}{\Omega_{B-}-\Omega}+\frac{\Omega}{\Omega_{B+}+\Omega}\right], \\
& c_{\omega}\left(\varepsilon_{3}-\varepsilon_{4}\right) \Omega=\frac{q c_{\omega}}{\left(m_{+}+m_{-}\right)}\left[\frac{\Omega}{\Omega-\Omega_{B+}}-\frac{\Omega}{\Omega+\Omega_{B-}}\right] .
\end{aligned}
$$

The results of Eqs. (4.11)-(4.14) demonstrate, once more, that in the high-frequency limit of the spectrum the magnetic current dominates against the vortical current. For intermediate frequencies (i.e. as soon as we reduce $\Omega$ ) the terms containing the natural frequencies of the plasma come then into play so that for the $R$ - and $L$-modes the corresponding dispersion relations become

$$
\begin{array}{ll}
\Omega^{2}=k^{2}+k c_{B}+\frac{\Omega_{p}^{2} \Omega}{\left(\Omega-\Omega_{B-}\right)}, & R \text { - mode, } \\
\Omega^{2}=k^{2}-k c_{B}+\frac{\Omega_{p}^{2} \Omega}{\left(\Omega+\Omega_{B-}\right)}, & L-\text { mode. }
\end{array}
$$

As in the standard case, the phase velocity of the $R$-mode is greater than that of the $L$-mode. In Eq. (4.15) we assumed $m_{+}>m_{-}$, and therefore, $\Omega_{B+}<\Omega_{B-}$. In the limit $k \rightarrow 0$ the $R$-mode cutoff occurs above $\Omega_{p}$ while the $L$-mode cutoff occurs below $\Omega_{p}$ (i.e., recalling Eqs. (4.6) and (4.7), $\Omega \rightarrow \Omega_{R}$ and $\left.\Omega \rightarrow \Omega_{L}\right)$. In the low-frequency limit $\varepsilon_{R}$ and $\varepsilon_{L}$ coincide to leading order in $\left(\Omega / \Omega_{B+}\right)$ and in $\left(\Omega / \Omega_{B-}\right)$ since

$$
\begin{aligned}
\lim _{\Omega \rightarrow 0} \varepsilon_{R}(\Omega) & =\lim _{\Omega \rightarrow 0} \varepsilon_{L}(\Omega) \rightarrow 1+\frac{\Omega_{p}^{2}}{\Omega_{B+} \Omega_{B-}}=1+\frac{1}{v_{A}^{2}}, \\
v_{A} & =\frac{B_{0}}{\sqrt{4 \pi n_{0}\left(m_{+}+m_{-}\right)}},
\end{aligned}
$$

\footnotetext{
${ }^{12}$ This limit is formal in the sense that $\Omega$ can get larger than all the other frequencies of the problem but always remain smaller than the plasma frequency $\Omega_{p}$.
}

where $v_{A}$ denotes the Alfvén velocity of the system. In the low-frequency limit the dispersion relations for the $R$-mode and for the $L$-mode are, respectively,

$$
\begin{aligned}
& \Omega^{2}=\frac{v_{A}^{2}}{1+v_{A}^{2}}\left\{k^{2}+k\left[c_{B}+\frac{q c_{\omega}}{m}\left(\frac{\Omega}{\Omega_{B+}}+\frac{\Omega}{\Omega_{B-}}\right)\right]\right\}, \\
& \Omega^{2}=\frac{v_{A}^{2}}{1+v_{A}^{2}}\left\{k^{2}-k\left[c_{B}-\frac{q c_{\omega}}{m}\left(\frac{\Omega}{\Omega_{B+}}+\frac{\Omega}{\Omega_{B-}}\right)\right]\right\},
\end{aligned}
$$

since $v_{A} \ll 1$ we also have $v_{A}^{2} /\left(1+v_{A}^{2}\right) \simeq v_{A}^{2}$.

Having determined the dispersion relations in the case of parallel propagation, the Faraday rotation rate can be easily determined with the standard procedure. The generalized Faraday rotation angle experienced by the linearly polarized radiation traveling parallel to the magnetic field direction can be obtained as

$$
\begin{aligned}
\Delta \Phi= & \frac{\Omega}{2}\left\{\frac{c_{B}}{\Omega}+c_{\omega} \varepsilon_{3}+\sqrt{\varepsilon_{L}+\left[\frac{c_{B}}{2 \Omega}+\frac{c_{\omega}}{2}\left(\varepsilon_{3}+\varepsilon_{4}\right)\right]^{2}}\right. \\
& \left.-\sqrt{\varepsilon_{R}+\left[\frac{c_{B}}{2 \Omega}+\frac{c_{\omega}}{2}\left(\varepsilon_{3}-\varepsilon_{4}\right)\right]^{2}}\right\} \Delta \mathrm{L}
\end{aligned}
$$

where $\Delta \mathrm{L}$ is the distance traveled by the signal in the direction parallel to the magnetic field direction. It is interesting to compare the contribution of the terms depending upon $c_{B}$ and those depending upon the background magnetic field intensity, i.e. the terms appearing in the squared brackets. Recalling the expressions of $\left(\varepsilon_{R}, \varepsilon_{L}\right)$ we have that $\Omega_{B+} \ll \Omega_{B-}$ and $\Omega_{p+} \ll \Omega_{p-}$ (always assuming $\left.{ }^{13} m_{+} \gg m_{-}\right)$. In this case $\Delta \Phi / \Delta L$ interpolates between the standard result $\left(\Omega_{B-} / 2\right)\left(\Omega_{p_{-}} / \Omega\right)^{2}$ (valid when $c_{B} \rightarrow 0$ ) and the constant rotation rate $c_{B} / 2$ (valid when $B_{0} \rightarrow 0$ as in the case of free-field propagation). As it can be explicitly verified the $c_{\omega}$ is subdominant at high frequencies and can be neglected.

\section{Orthogonal propagation}

By setting $\theta \rightarrow \pi / 2$ in Eq. (4.1) and recalling the results of Eq. (A7) we obtain the following simple equation:

$$
\begin{aligned}
& n^{4}\left(\varepsilon_{L}+\varepsilon_{R}\right) \Omega^{2}-n^{2}\left\{c_{B}^{2}\left(\varepsilon_{L}+\varepsilon_{R}\right)+c_{B} c_{\omega}\left[\varepsilon_{4}\left(-\varepsilon_{L}+\varepsilon_{R}\right)\right.\right. \\
& \left.\left.\quad+\varepsilon_{3}\left(\varepsilon_{L}+\varepsilon_{R}\right)\right] \Omega+\left[\varepsilon_{\|} \varepsilon_{R}+\varepsilon_{L}\left(\varepsilon_{\|}+2 \varepsilon_{R}\right)\right] \Omega^{2}\right\} \\
& \quad+2 \varepsilon_{L} \varepsilon_{R} \Omega^{2} \varepsilon_{\|}=0 .
\end{aligned}
$$

\footnotetext{
${ }^{13}$ The hierarchy $m_{+} \gg m_{-}$has been privileged since it is the same hierarchy of ordinary electromagnetic plasmas where $m_{+}$ coincides with the mass of the ions (or protons) and $m_{-}$coincides with the mass of the electrons.
} 
The solution of Eq. (4.20) can be obtained by first solving in terms of $n^{2}$. The result is

$$
\begin{gathered}
n^{2}=\frac{\mathcal{J}(\varepsilon, \Omega) \pm \sqrt{\mathcal{M}(\varepsilon, \Omega)}}{2\left(\varepsilon_{R}+\varepsilon_{L}\right) \Omega^{2}} \\
\mathcal{J}(\varepsilon, \Omega)=c_{B}^{2}\left(\varepsilon_{L}+\varepsilon_{R}\right)+c_{B} c_{\omega}\left[\varepsilon_{4}\left(-\varepsilon_{L}+\varepsilon_{R}\right)\right. \\
\left.+\varepsilon_{3}\left(\varepsilon_{L}+\varepsilon_{R}\right)\right] \Omega+\left[\varepsilon_{\|} \varepsilon_{R}+\varepsilon_{L}\left(\varepsilon_{\|}+2 \varepsilon_{R}\right)\right] \Omega^{2}
\end{gathered}
$$

$$
\begin{aligned}
\mathcal{M}(\varepsilon, \Omega)= & -8 \varepsilon_{\|} \varepsilon_{R} \varepsilon_{L}\left(\varepsilon_{L}+\varepsilon_{R}\right) \Omega^{4}+\left\{c_{B}^{2}\left(\varepsilon_{L}+\varepsilon_{R}\right)\right. \\
& +c_{B} c_{\omega}\left[\varepsilon_{4}\left(\varepsilon_{R}-\varepsilon_{L}\right)+\varepsilon_{3}\left(\varepsilon_{L}+\varepsilon_{R}\right)\right] \Omega \\
& \left.+\left[\varepsilon_{\|} \varepsilon_{R}+\varepsilon_{L}\left(\varepsilon_{\|}+2 \varepsilon_{R}\right)\right] \Omega^{2}\right\}^{2}
\end{aligned}
$$

Equation (4.21) in the limit $c_{\omega} \rightarrow 0$ and $c_{B} \rightarrow 0$ reduces to the ordinary mode if we choose the plus (i.e. $n^{2}=\varepsilon_{\|}$) and to the extraordinary mode [i.e. $\left.n^{2}=2 \varepsilon_{\mathrm{R}} \varepsilon_{\mathrm{L}} /\left(\varepsilon_{\mathrm{R}}+\varepsilon_{\mathrm{L}}\right)\right]$ if we choose the minus. In the high-frequency limit the terms multiplying the vortical current are always negligible as already remarked in the case of the parallel propagation. The phenomena related to the oblique propagation are not be specifically discussed.

\section{Spectrum around a hypermagnetic knot}

Introducing the three mutually orthogonal unit vectors $\hat{a}(z, p), \hat{b}(z, p)$ and $\hat{z}$ defined in Appendix $\mathrm{B}$, we can consider the modes of fluctuation of the hypermagnetic field around a fully inhomogeneous background $\vec{B}_{0}(t, \vec{x})$, namely

$$
\vec{B}(t, \vec{x})=\vec{B}_{0}(t, \vec{x})+\delta \vec{B}(t, \vec{x}) .
$$

Since the background solution is not uniform we can align $\vec{B}_{0}$ along $\hat{a}(z, p)$ and write that $\vec{B}_{0}(z)=B_{0} \hat{a}(z, p)$. The background equations are solved by setting $p=-c_{B}$ (since $\vec{\nabla} \times \vec{B}_{0}=p \vec{B}_{0}$ ). As in the homogeneous case the velocities vanish on the background solution. For $L<1 / c_{B}$ the background field is homogeneous, and the previous analyses apply. For typical length scales larger than the scale of spatial variation of hypermagnetic knot (i.e. $L \gg 1 / c_{B}$ ) there are two separate possibilities for the perturbed velocity field: either $\delta \vec{v} \| \vec{B}_{0}$ or $\delta \vec{v} \perp \vec{B}_{0}$. These two cases now are separately examined.

The case of parallel propagation mirrors exactly the one already discussed in the case of uniform field. If we assume that $\delta \vec{v} \| \vec{B}_{0}$ the dispersion relations follow from

$$
\left\{\left[k^{2}-\Omega^{2} \varepsilon_{\|}(\Omega)\right]^{2}-c_{B}^{2} k^{2}\right\} \varepsilon_{\|}(\Omega)=0 .
$$

The parallel dielectric tensor is $\varepsilon_{\|}(\Omega)=1-\Omega_{p}^{2} /\left[\Omega\left(\Omega+i \Gamma_{c}\right)\right]$ where the correction coming from the collision rate has been added for immediate convenience. The solution $\varepsilon_{\|}(\Omega)=0$ gives, as before, the electrostatic wave. The solution of $\left\{\left[k^{2}-\Omega^{2} \varepsilon_{\|}(\Omega)\right]^{2}-c_{B}^{2} k^{2}\right\}=0$ gives, respectively, a highfrequency and a low-frequency branch. The high-frequency branch has the same dispersion relation of the free-field case, namely $\Omega^{2} \simeq \Omega_{p}^{2}+k^{2} \mp k c_{B}$. The low-frequency branch is instead derived from the explicit form of the dispersion relation written as

$$
\Omega+i \Gamma_{c}=\frac{\Omega^{2}}{k^{2}}\left(\Omega+i \Gamma_{c}\right)-\frac{\Omega_{p}^{2} \Omega}{k^{2}} \pm \frac{c_{B}}{k}\left(\Omega+i \Gamma_{c}\right) ;
$$

neglecting the first term at the right-hand side of the previous equation (which is unimportant at low frequencies) we have that

$$
\Omega=-\frac{i \Gamma_{c}\left(1 \mp c_{B} / k\right)}{1+\Omega_{p}^{2} / k^{2} \mp c_{B} / k} .
$$

The low-frequency mode, in which the conducting current dominates over the displacement current, has no counterpart in vacuum. In the low-frequency mode, a small electric field proportional to $\Gamma_{c}$ exists to give the necessary current parallel to the magnetic field. In the limit $\Gamma_{c} \rightarrow 0$ both the electric field and resistivity vanish, and the lowfrequency mode becomes the force-free field. As expected the same phenomenon occurs in the absence of magnetic and vortical currents $[18,19]$.

In the case of orthogonal propagation the fluctuations of the hypermagnetic field and of the velocity can be decomposed by using the gyrotropic basis of Appendix B:

$$
\begin{aligned}
\delta \vec{B}(t, z) & =\delta B_{1}(t) \hat{b}(z, p)+\delta B_{2}(t) \hat{z}, \\
\delta \vec{v}^{( \pm)}(t, z) & =\delta v_{1}^{( \pm)}(t) \hat{b}(z, p)+\delta v_{2}^{( \pm)}(t) \hat{z} .
\end{aligned}
$$

For a generic velocity fluctuation orthogonal to $\vec{B}_{0}$ we have $\delta \vec{v} \times \vec{B}_{0}=\left[B_{0}(\delta \vec{v} \cdot \hat{z}) \hat{b}-B_{0}(\delta \vec{v} \cdot \hat{b}) \hat{z}\right]$; the solutions for $\delta v_{1}^{( \pm)}(t)$ and $\delta v_{2}^{( \pm)}(t)$ can then be expressed as

$$
\begin{aligned}
& \delta v_{1}^{( \pm)}(\Omega)=\frac{q}{m_{ \pm}\left(\Omega_{B \pm}^{2}-\Omega^{2}\right)}\left[ \pm i \Omega \delta E_{1}+\Omega_{B \pm} \delta E_{2}\right], \\
& \delta v_{2}^{( \pm)}(\Omega)=\frac{q}{m_{ \pm}\left(\Omega_{B \pm}^{2}-\Omega^{2}\right)}\left[ \pm i \Omega \delta E_{2}-\Omega_{B \pm} \delta E_{1}\right] .
\end{aligned}
$$

The dispersion relations in this case are given by

$$
\varepsilon_{R} \varepsilon_{L}-\frac{c_{B} c_{\omega}}{2 \Omega}\left[\varepsilon_{L}\left(\varepsilon_{3}-\varepsilon_{4}\right)+\varepsilon_{R}\left(\varepsilon_{3}+\varepsilon_{4}\right)\right]=0 .
$$

In the high-frequency limits ${ }^{14}$ defined by Eqs. (4.13) and (4.14), Eq. (4.30) is satisfied if $\varepsilon_{R} \varepsilon_{L}=0$ which is verified

\footnotetext{
${ }^{14}$ The remark on the formal sense of the infinite frequency limit discussed prior to Eqs. (4.13) and (4.14) holds also for Eq. (4.30) evaluated in the limit $\Omega \rightarrow \infty$.
} 
when either $\varepsilon_{L}$ or $\varepsilon_{R}$ are vanishing. Equation (4.30) leads to vanishing group velocity in the high-frequency regime: The corresponding modes are then not propagating. The proper frequencies defined by these equations have been already derived in Eqs. (4.11) and (4.12).

\section{SINGLE FLUID DESCRIPTION AND ITS IMPLICATIONS}

The two-fluid equations can now be combined with the purpose of deriving the effective single fluid description valid for sufficiently large length scales and for frequencies much smaller than $\Omega_{p}$ and $\Omega_{B \pm}$. The one-fluid variables are the total current $\vec{J}=q\left(n_{+}, \vec{v}_{+}-n_{-} \vec{v}_{-}\right)$, the bulk velocity of the plasma $\vec{v}=\left(m_{+} \vec{v}_{+}+m_{-} \vec{v}_{-}\right) /\left(m_{+}+m_{-}\right)$and the total mass density $\rho_{m}=\left(m_{+} n_{+}+m_{-} n_{-}\right)$. In the globally neutral case $\vec{J}$ and $\rho_{m}$ become, respectively, $\vec{J}=n_{0}\left(\vec{v}_{+}-\right.$ $\left.\vec{v}_{-}\right)$and $\rho_{m}=n_{0}\left(m_{+}+m_{-}\right)$. Summing up Eq. (3.9) (multiplied by $m_{+}$) and Eq. (3.10) (multiplied by $m_{-}$) the evolution equation for the bulk velocity of the plasma is

$$
\rho_{m}\left[\partial_{t} \vec{v}+\vec{v} \cdot \vec{\nabla} \vec{v}\right]=\vec{J} \times \vec{B}-\vec{\nabla} P+\eta \nabla^{2} \vec{v},
$$

where the shear viscosity contribution, labeled by $\eta$, has been added for convenience. ${ }^{15}$ Taking the difference of Eqs. (3.9) and (3.10) (and assuming $m_{+}>m_{-}$) the generalized Ohm's law can be written as

$$
\partial_{t} \vec{J}+\Gamma_{c} \vec{J} \simeq \frac{\Omega_{P-}^{2}}{4 \pi}\left(\vec{E}+\vec{v} \times \vec{B}+\frac{\vec{\nabla} p_{-}}{q n_{0}}-\frac{\vec{J} \times \vec{B}}{q n_{0}}\right),
$$

where global neutrality has been assumed. Note that we have also kept the thermoelectric term (depending on the pressure gradient of the lightest charge carriers) and the Hall term. Since we mainly consider the case of homogeneous pressures the thermoelectric term is neglected; the Hall term is a higher order contribution, as we argue here.

In the globally neutral case the single fluid equations stipulate that $\vec{E}, \vec{B}$ and $\vec{J}$ are all solenoidal (i.e. $\vec{\nabla} \cdot \vec{E}=$ $\vec{\nabla} \cdot \vec{B}=\vec{\nabla} \cdot \vec{J}=0$ ). A fourth possible solenoidal vector is the bulk velocity of the plasma $\vec{v}$. Indeed, since the evolution of $\rho_{m}$ and $\rho_{q}=q\left(n_{+}-n_{-}\right)$is given by ${ }^{16}$

$$
\partial_{t} \rho_{m}+\vec{\nabla} \cdot\left(\rho_{m} \vec{v}\right)=0, \quad \partial_{t} \rho_{q}+\vec{\nabla} \cdot \vec{J}=0,
$$

the incompressible closure $\vec{\nabla} \cdot \vec{v}=0$ is adopted; consistent with the incompressible closure $\rho_{m}$ is considered

\footnotetext{
${ }^{15}$ If the total pressure does not vanish Eq. (5.1) is modified as follows $\partial_{t}[w \vec{v}]+(\vec{v} \cdot \vec{\nabla})[w \vec{v}]+\vec{v} \vec{\nabla} \cdot[w \vec{v}]=-\vec{\nabla} P+\vec{J} \times \vec{B}+\eta \nabla^{2} \vec{v}$ where $w$, as already discussed, is the enthalpy density.

${ }^{16}$ Note that the global neutrality implies $\rho_{q}=0$, and Eq. (5.3) demands $\vec{\nabla} \cdot \vec{J}=0$ in full agreement with the solenoidal nature of the total Ohmic current.
}

homogeneous, at least in the first part of this section. A full discussion of other possible closures (such as the ones conventionally adopted in conventional plasmas) is desirable but beyond the scoped of this analysis. The remaining one-fluid equation containing the vortical and the magnetic currents, can be written as

$$
\vec{\nabla} \times \vec{B}-\partial_{t} \vec{E}=4 \pi \vec{J}+c_{\omega} \vec{\omega}-c_{B} \vec{B}, \quad \vec{\omega}=\vec{\nabla} \times \vec{v} .
$$

Since the one-fluid description involves the lowest branch of the spectrum we can neglect the displacement current that becomes relevant only for the electromagnetic propagation. For the same reason we can neglect the time derivative in Eq. (5.2), i.e. $\partial_{t} \vec{J} \ll \Omega_{p}^{2} \vec{E}$. Consequently Eqs. (5.2) and (5.4) in the low-frequency branch of the spectrum become

$$
\vec{\nabla} \times \vec{B}=4 \pi \vec{J}+c_{\omega} \vec{\omega}-c_{B} \vec{B}, \quad \vec{E}=\vec{J} / \sigma-\vec{v} \times \vec{B}
$$

Recalling that $\vec{\nabla} \times \vec{E}=-\partial_{t} \vec{B}$, Eq. (5.5) can be used to obtain an equation that is reminiscent of the magnetic diffusivity equation, namely

$$
\begin{aligned}
\partial_{t} \vec{B}= & \vec{\nabla} \times(\vec{v} \times \vec{B})+\frac{\nabla^{2} \vec{B}}{4 \pi \sigma}+\frac{1}{4 \pi \sigma} \vec{\nabla} \times\left(c_{\omega} \vec{\omega}\right) \\
& -\frac{1}{4 \pi \sigma} \vec{\nabla} \times\left(c_{B} \vec{B}\right) .
\end{aligned}
$$

Introducing the vorticity $\vec{\omega}$ into Eq. (5.1) and dividing both sides of the equation by $\rho_{m}$ we obtain

$$
\partial_{t} \vec{v}+\vec{\omega} \times \vec{v}=\frac{\vec{J} \times \vec{B}}{\rho_{m}}-\vec{\nabla}\left[\frac{P}{\rho_{m}}+\frac{v^{2}}{2}\right]+\nu_{k i n} \nabla^{2} \vec{v}
$$

Taking the curl of Eq. (5.7) the evolution equation of the vorticity becomes

$$
\partial_{t} \vec{\omega}=\vec{\nabla} \times(\vec{v} \times \vec{\omega})+\frac{\vec{\nabla} \times(\vec{J} \times \vec{B})}{\rho_{m}}+\nu_{k i n} \nabla^{2} \vec{\omega} .
$$

The most interesting solutions of the one-fluid equations will involve the situations where the vortical and the magnetic currents play the dominant role. However, before turning the attention on these classes solutions it is useful to remark that the equilibrium solutions of the plasma at rest (i.e. $\vec{v}=0$ ) are simply given by

$$
\vec{\nabla} P=\vec{J} \times \vec{B}, \quad 4 \pi \vec{J}=\left(\vec{\nabla} \times \vec{B}-c_{\omega} \vec{\omega}+c_{B} \vec{B}\right) .
$$

From Eq. (5.9) it is immediate to obtain the following three identities: 


$$
4 \pi \vec{\nabla} P=(\vec{\nabla} \times \vec{B}) \times \vec{B}, \quad(\vec{B} \cdot \vec{\nabla}) P=0,
$$

$(\vec{J} \cdot \vec{\nabla}) P=0$.

The relations of Eq. (5.10) are not explicitly modified by the presence of vortical and magnetic currents. The two last conditions in Eq. (5.10) define the so-called magnetic surfaces: The pressure gradient vanishes along the lines of magnetic force and along the current lines. We conclude that neither the vortical nor the magnetic current affect directly the equilibrium solutions.

\section{A. Bulk velocity parallel to the magnetic field direction}

The single fluid AMHD equations admit various solutions that have no counterpart in the case of ordinary MHD. Consider first the situation where the hypermagnetic magnetic field and the velocity are parallel and have nonvanishing magnetic and kinetic gyrotropy, i.e.

$$
\begin{aligned}
\vec{v} \times \vec{B} & =0, \quad \vec{v} \cdot \vec{\nabla} \times \vec{v}=p_{v}(t) v^{2}, \\
\vec{B} \cdot \vec{\nabla} \times \vec{B} & =p_{B}(t) B^{2} .
\end{aligned}
$$

The simplest way to realize the situation described by Eq. (5.11) is to require that $\vec{v}$ and $\vec{B}$ are both Beltrami-like fields (see Appendix B for this terminology) characterized by $\vec{\nabla} \times \vec{v}=p_{v}(t) \vec{v}$ and $\vec{\nabla} \times \vec{B}=p_{B}(t) \vec{B}$. Moreover, since $\vec{v} \times \vec{B}=0$, it is natural ${ }^{17}$ to require that $p_{v}(t)=p_{B}(t)$. From Eq. (5.5) the total current $\vec{J}$ can be easily determined; the Ohmic electric field is then given by

$$
\vec{E}=\frac{p_{B}(t)+c_{B}(t)}{4 \pi \sigma} \vec{B}-\frac{c_{\omega}(t)}{4 \pi \sigma} \vec{\omega} .
$$

From Eq. (5.6) the hypermagnetic field is obtained by solving the following equation:

$$
\partial_{t} \vec{B}=-\frac{p_{B}(t)\left[p_{B}(t)+c_{B}(t)\right]}{4 \pi \sigma(t)} \vec{B}+\frac{c_{\omega}(t)}{4 \pi \sigma(t)} p_{B}(t) \vec{\omega},
$$

where $\vec{\omega}(t, z)$ is the solution of Eq. (5.8). Thanks to the symmetries of the problem the solution of this equation is given by $\vec{\omega}(t, z)=\vec{\omega}_{0}(z) \exp \left[-\int_{0}^{t} p_{B}^{2}\left(t^{\prime}\right) \nu_{k i n}\left(t^{\prime}\right) d t^{\prime}\right]$ where $\omega_{0}(z)$ is the initial vorticity which can also be written as $\vec{\omega}_{0}(z)=p_{B}(0) \vec{v}_{0}(z)$. Equation (5.13) can then be solved in general terms. However, recalling that $c_{B}(t)$ and $c_{\omega}(t)$ are explicit functions of time they depend on the rescaled

\footnotetext{
${ }^{17}$ To impose $p_{B}=p_{v}$ means that the inhomogeneity scale of the magnetic and kinetic knots are the same. According to some this condition is natural; according to some others it is just practical since in this case the system can be solved exactly.
}

chemical potential, ${ }^{18}$ and since $p_{B}(t)$ is arbitrary we can choose $p_{B}(t)=-c_{B}(t)$. In this case, the solution of Eq. (5.13) shares the same properties of the general solution, but it is mathematically simpler:

$\vec{B}(t, z)=\vec{\omega}_{0}(z) \int_{0}^{t} d t^{\prime} \frac{c_{B}\left(t^{\prime}\right) c_{\omega}\left(t^{\prime}\right)}{4 \pi \sigma\left(t^{\prime}\right)} e^{-\int_{0}^{t^{\prime}} c_{B}^{2}\left(t^{\prime \prime}\right) \nu_{k i n}\left(t^{\prime \prime}\right) d t^{\prime \prime}}$.

The results of Eq. (5.14) describe the generation of the hypermagnetic field thanks to some initial vortical current. To deepen this question let us assume that $c_{\omega}$ and $c_{B}$ are both constant. Equation (5.14) can then be solved, and the result is

$$
\vec{B}(z, t)=-\frac{\vec{v}_{0}(z) c_{\omega}}{4 \pi \nu_{k i n} \sigma}\left[1-e^{-\nu_{k i n} c_{B}^{2} t}\right],
$$

where we used that $\vec{\omega}_{0}(z)=-c_{B} \vec{v}_{0}(z)$ when $c_{B}$ is constant in time and $c_{B}(0)=c_{B}$. This result is also valid for a relativistic equation of state (i.e. $w=4 \rho / 3$ ) provided the incompressible closure is consistently adopted and can be easily generalized to curved backgrounds. While these generalizations are not germane to our theme it is worth emphasizing that in the limit $t \rightarrow \infty$ the suppression of the magnetic field is controlled by $4 \pi \sigma \nu_{k i n}$ which is nothing but the Prandtl number given as the ratio of the magnetic and of the kinetic Reynolds number [2]. The Prandtl number is roughly independent on the temperature. For instance, at the electroweak epoch [10] we would have that $\nu_{\text {kin }} \simeq$ $1 /\left(\alpha^{\prime 2} T\right)$ while $\sigma \simeq T / \alpha^{\prime}$ where $\alpha^{\prime}=g^{\prime 2} /(4 \pi)$. Recalling the results of Eqs. (2.19) and (2.20) we therefore have that

$$
\lim _{t \rightarrow \infty} \vec{B}(t, z)=\frac{c_{\omega}(T)}{4 \pi} \alpha^{\prime 3} \vec{v}_{0}, \quad c_{\omega}(T)=2 T^{2} a\left(\bar{\mu}_{R}\right),
$$

where $a_{B}\left(\bar{\mu}_{R}\right)$ is the usual arbitrary function of the rescaled chemical potential $\bar{\mu}_{R}=\mu_{R} / T$ [see Eqs. (2.19) and (2.20)]. The suppression due to the conductivity is therefore eliminated, and what is left is a milder suppression $\mathcal{O}\left(\alpha^{3}\right)$. In the same limit the hypermagnetic current turns out to be more suppressed than the vortical current. As long as the inverse of the Prandtl number scales as $\alpha^{\prime 3}$ the previous discussion is generally valid, and this is what happens in the case of the electroweak plasma [10] (see also [20] for specific estimates of the conductivity in the electroweak phase ${ }^{19}$ ).

\footnotetext{
${ }^{18}$ In this discussion we keep the time dependence in the kinetic coefficients even if, strictly speaking, $c_{B}$ and $c_{\omega}$ may depend on the temperature and on the chemical potential, but they are constant in time. However, in curved backgrounds a mild breaking of conformal invariance may induce a time dependence which is, however, not central to the present analysis.

${ }^{19}$ Similar kinds of considerations can also be developed in the case of a strongly interacting plasma as long as the same scaling occurs [21].
} 


\section{B. Bulk velocity orthogonal to the magnetic field direction}

If the hypermagnetic field and the bulk velocity of the plasma are orthogonal (i.e. $\vec{v} \cdot \vec{B}=0$ ), employing the helical basis of Appendix B the hypermagnetic field and the velocity field can be written as

$$
\begin{aligned}
\vec{B}(t, z) & =B_{0}(t) \hat{z}+B_{1}(t) \hat{a}(z, p), \\
\vec{v}(z, t) & =v(t) \hat{b}(z, p) .
\end{aligned}
$$

From Eq. (5.5) the AMHD current becomes

$$
\begin{aligned}
\vec{J}(t, z)= & \frac{B_{1}(t)}{4 \pi}\left[c_{B}+p\right] \hat{a}(z, p)-\frac{c_{\omega}}{4 \pi} p v(t) \hat{b}(z, p) \\
& +\frac{c_{B} B_{0}(t)}{4 \pi} \hat{z} .
\end{aligned}
$$

By analyzing the structure of the evolution equation of the magnetic field and of the vorticity it emerges that the system is consistent provided $\partial_{t} B_{0}=0$ (i.e. constant magnetic field along $\hat{z}$ ) and provided $c_{\omega}=0$. In this case the coupled evolution of the vorticity and of the magnetic field obeys

$$
\begin{aligned}
\frac{d \omega}{d t} & =\frac{p c_{B} B_{0}}{4 \pi \rho_{m}} B_{1}-\frac{B_{0}}{4 \pi \rho_{m}} p\left[c_{B}+p\right] B_{1}, \\
\frac{d B_{1}}{d t} & =\omega B_{0}-\frac{p B_{1}}{4 \pi \sigma}\left[c_{B}+p\right],
\end{aligned}
$$

where $\omega(t)=p v(t)$ and $\omega(z, t)=\omega(t) \hat{b}(z, p)$. The equations can be diagonalized with a specific choice of the coordinate system. The simplest and most convenient one is $p=-c_{B}$; in this case the two equations can be combined by differentiating once Eq. (5.19). The result is

$$
\frac{d^{2} B_{1}}{d t^{2}}+c_{B}^{2} v_{A}^{2} B_{1}=0, \quad v_{A}=\frac{B_{0}}{\sqrt{4 \pi \rho_{m}}},
$$

where $v_{A}$ is the Alfvén velocity. This solution has been swiftly presented in Ref. [7] and recently rediscovered in [22]. Equation (5.20) describes the AMHD analog of the nonlinear Alfvén wave. The anomalous Alfvén wave has been already discussed in Sec. III as a low-frequency limit of the two-fluid equations.

\section{Fully nonlinear evolution and baryogenesis}

So far we considered small fluctuations of the chiral concentration around an otherwise homogeneous value denoted by $n_{1}$ in Sec. II. In the opposite case the AMHD equations imply a specific relation between the concentration (or the chemical potential) and the topological properties of the hypermagnetic fields. To illustrate this point we show that close to equilibrium the chemical potential is determined not only by the magnetic gyrotropy but also by the total vorticity of the plasma. Hypermagnetic field configurations with nonvanishing gyrotropy have been used to model the generation of the baryon or lepton asymmetry [10] (see also [23-25]). Consider, therefore, the evolution equation of the chemical potential which can be written as

$$
\partial_{t} \bar{\mu}_{R}+\Gamma \bar{\mu}_{R}=-\frac{4 \mu_{0}}{\varsigma} \mathcal{A}_{R} \vec{E} \cdot \vec{B},
$$

where $\Gamma$ is the perturbative rate of he chirality flip processes (in the case of [10] it is determined by the scattering of right electrons with the Higgs and gauge bosons and with the top quarks because of their large Yukawa coupling). In Eq. (5.21) we also used the following general relation relation $\bar{\mu}_{R}=\mu_{0} n_{R} / \varsigma$ where $\mu_{0}$ is a numerical factor depending on the specific features of the plasma while $\varsigma$ is, as usual, the entropy density.

To compute $\bar{\mu}_{R}$ in the proximity of an equilibrium situation we need to deduce the hyperelectric field. Recalling then Eq. (5.5), the hyperelectric field can be related to the total Ohmic current so that Eq. (5.21) becomes

$$
\begin{gathered}
\partial_{t} \bar{\mu}_{R}+\Gamma \bar{\mu}_{R}-\frac{8 T^{2} a_{B}\left(\bar{\mu}_{R}\right) \mu_{0}}{\sigma \varsigma} \mathcal{A}_{R} \vec{\omega} \cdot \vec{B}+16 \frac{\mu_{0} T \bar{\mu}_{R}}{\sigma \varsigma} \mathcal{A}_{R}^{2} B^{2} \\
=-\frac{\mu_{0}}{\pi \sigma \varsigma} \vec{B} \cdot \vec{\nabla} \times \vec{B}
\end{gathered}
$$

We now choose $a_{B}\left(\bar{\mu}_{R}\right)=\bar{\mu}_{R}$; if $a_{B}\left(\bar{\mu}_{R}\right) \neq \bar{\mu}_{R}$ in the evolution equation of the concentration we should add a further term proportional to $\vec{\nabla} \cdot\left[c_{R B}\left(\bar{\mu}_{R}\right) \vec{B}\right]$. This term vanishes in the case $a_{B}\left(\bar{\mu}_{R}\right) \propto \bar{\mu}_{R}: c_{R B}\left(\bar{\mu}_{R}\right)$ contains the derivative of $a_{B}\left(\bar{\mu}_{R}\right)$ with respect to $\bar{\mu}_{R}$, and it is therefore constant. In more general situations Eq. (5.22) just contains a supplementary contribution of the type $\vec{B} \cdot \vec{\nabla} \bar{\mu}_{R}$.

The rescaled chemical potential enters the infinitely conducting limit (see Appendix B), and the smallness of the particle asymmetries is the rationale for the minuteness of the rescaled chemical potentials in approximate thermal equilibrium. At equilibrium, we can determine $\bar{\mu}_{R}$ from Eq. (5.22), and the result is given by

$$
\bar{\mu}_{R}=-\left(\frac{\mu_{0} \mathcal{A}_{R}}{\pi \varsigma \sigma}\right) \frac{\vec{B} \cdot \vec{\nabla} \times \vec{B}}{\left[\Gamma+\Gamma_{B}-\Gamma_{\omega}\right]} .
$$

While $\Gamma$ is the perturbative chirality flip rate, the other terms can be understood as rates stemming from the hypermagnetic current and from the vortical current, and they are

$$
\Gamma_{B}=\frac{16 \mu_{0}}{\varsigma \sigma} \mathcal{A}_{R}^{2} T B^{2}, \quad \Gamma_{\omega}=\frac{8 T^{2} \mu_{0}}{\pi \sigma \varsigma} \mathcal{A}_{R} \vec{\omega} \cdot \vec{B}
$$


In the case of right electrons (see [10]) $\mathcal{A}_{R}=$ $-g^{\prime 2} y_{R}^{2} /\left(64 \pi^{2}\right)$ where $g^{\prime}$ denotes the gauge coupling and $y_{R}=-2$ is the hypercharge assignment of the right electrons. In the same situation we have $\bar{\mu}_{R}=\mu_{0} n_{R} / \varsigma$ and $\mu_{0}=87 \pi^{2} N_{\text {eff }} / 220$, where $N_{\text {eff }}$ is the effective number of relativistic degrees of freedom of the system. ${ }^{20}$ If the plasma is hypercharge neutral the value of the chemical potential can be estimated from the asymmetry in the case where all the standard model charges are in complete thermal equilibrium. If all the asymmetry is attributed to the right electrons (which is, in some sense, the most favorable situation) then $\bar{\mu}_{R}=\left(87 \pi^{2} / 220\right) N_{\text {eff }}\left(n_{R} / \varsigma\right)$ where $N_{\text {eff }}=$ 106.75. With these specifications Eq. (5.23) becomes

$$
\bar{\mu}_{R}=-\frac{783 \alpha^{\prime}}{88 \pi \sigma T^{3}} \frac{\vec{B} \cdot \vec{\nabla} \times \vec{B}}{\left[\Gamma+\Gamma_{B}-\Gamma_{\omega}\right]}, \quad \Gamma_{B}=\frac{783 \alpha^{2}}{22 \pi^{2} \sigma} \frac{B^{2}}{T^{3}},
$$

where $\alpha^{\prime}=g^{\prime 2} /(4 \pi)$. Equation (5.25) coincides with the previous results (see e.g. Eq. (6.15) of the last paper of [10] and see also [23-25]) in the limit $\Gamma_{\omega} \rightarrow 0$. The results of Eq. (5.25) show that the final value of the chemical potential depends on the properties of the flow entering the definition of $\Gamma_{\omega}$. In summary we can say that the magnetic currents and the vortical currents can affect a number of processes such as the formation of the baryon asymmetry or the dynamics of the electroweak phase transition. Similar kinds of effects can be expected in the case of strongly interacting plasmas where the magnetic gyrotropy can also determine the properties of the chemical potential.

\section{CONCLUDING REMARKS}

The dispersion relations of anomalous magnetohydrodynamics are affected by the vortical and the hypermagnetic currents. The vortical currents do not impact on the high-frequency branch of the spectrum, but the opposite is true at lower frequencies where new solutions describe the simultaneous presence of hypermagnetic knots and fluid vortices. These parity-odd configurations carry, respectively, hypermagnetic and kinetic gyrotropy. The physical properties of the system roughly interpolate between the features of conventional chiral liquids, and the results are valid for cold electromagnetic plasmas. While chiral currents are anomalous and do not contribute to entropy production, vector currents are associated with the generalized Joule heating.

\footnotetext{
${ }^{20}$ There have been a number of suggestions for possible roles that the Abelian hypermagnetic Chern-Simons term might play in cosmology. One of them is related to the observation that righthanded electrons, which do not take part in weak interactions and also have a very small Yukawa coupling, are practically decoupled from the thermal ensemble above temperatures of about $10 \mathrm{TeV}$.
}

When chiral and Ohmic currents are simultaneously present the second law of thermodynamics constrains the kinetic coefficients. The hypermagnetic, vortical and Ohmic currents affect the evolution of the gauge fields and determine the hyperelectric field of the plasma. In anomalous magnetohydrodynamics the perfectly conducting limit is well posed, and the hypermagnetic helicity of the knots is strictly conserved, as it happens in the case of conventional plasmas. ${ }^{21}$ The hypermagnetic currents are then completely washed out in the perfectly conducting limit and strongly suppressed when the conductivity is large but finite. Close to thermal equilibrium the concentration of the chiral species and the corresponding chemical potential will depend not only on the hypermagnetic gyrotropy but also on the vortical currents.

In summary the evolution equations of anomalous magnetohydrodynamics offer a minimal theoretical framework where the interplay between conduction currents and chiral currents can be quantitatively analyzed. It is therefore fair to say that the results derived here complement and extend some of the present and earlier strategies aimed at an improved understanding of chiral liquids when generalized Ohmic effects cannot be neglected in the evolution of hypermagnetic and hyperelectric fields at finite fermionic density.

\section{APPENDIX A: GENERALIZED APPLETON- HARTREE EQUATION}

\section{Explicit form of $\varepsilon_{s}$ and $\varepsilon_{v}$}

We are going to give, in what follows, the explicit form of the dielectric tensors appearing in Sec. III. The matrix form of $\varepsilon_{s}(\Omega)$ and $\varepsilon_{v}(\Omega)$ is given by

$$
\begin{aligned}
& \varepsilon_{s}(\Omega)=\left(\begin{array}{ccc}
\varepsilon_{1}(\Omega) & i \varepsilon_{2}(\Omega) & 0 \\
-i \varepsilon_{2}(\Omega) & \varepsilon_{1}(\Omega) & 0 \\
0 & 0 & \varepsilon_{\|}(\Omega)
\end{array}\right), \\
& \varepsilon_{v}(\Omega)=\left(\begin{array}{ccc}
\varepsilon_{3}(\Omega) & -i \varepsilon_{4}(\Omega) & 0 \\
i \varepsilon_{4}(\Omega) & \varepsilon_{3}(\Omega) & 0 \\
0 & 0 & 0
\end{array}\right),
\end{aligned}
$$

\footnotetext{
${ }^{21}$ This point is very important, and it has been already emphasized in Ref. [7] by analyzing explicitly the conservation of the helicity. The key observation is that in the perfectly conducting limit the chiral magnetic and the chiral vortical terms disappear from the hypermagnetic diffusivity equation [see Eq. (5.6)]. In this situation it can be shown that the magnetic configurations minimizing the energy density with the constraint that the helicity be conserved coincide, in the perfectly conducting limit, with the ones obtainable in ideal magnetohydrodynamics where the anomalous currents are neglected. This result, coming from variational considerations, explains why it has been repeatedly observed throughout the years that the solutions of ordinary magnetohydrodynamics can be lifted to the anomalous case (see e.g. [10]) when the conductivity goes formally to infinity.
} 
where $\varepsilon_{1}(\Omega), \varepsilon_{2}(\Omega), \varepsilon_{3}(\Omega), \varepsilon_{4}(\Omega)$ and $\varepsilon_{\|}(\Omega)$ are defined as

$$
\begin{gathered}
\varepsilon_{1}(\Omega)=1-\frac{\Omega_{p+}^{2}}{\Omega^{2}-\Omega_{B+}^{2}}-\frac{\Omega_{p-}^{2}}{\Omega^{2}-\Omega_{B-}^{2}}, \\
\varepsilon_{2}(\Omega)=\left(\frac{\Omega_{B-}}{\Omega}\right) \frac{\Omega_{p-}^{2}}{\Omega^{2}-\Omega_{B-}^{2}}-\left(\frac{\Omega_{B+}}{\Omega}\right) \frac{\Omega_{p+}^{2}}{\Omega^{2}-\Omega_{B+}^{2}} . \\
\varepsilon_{\|}(\Omega)=1-\frac{\Omega_{p+}^{2}}{\Omega^{2}}-\frac{\Omega_{p-}^{2}}{\Omega^{2}}, \\
\varepsilon_{3}(\Omega)=\frac{q \Omega}{\left(m_{+}+m_{-}\right)}\left[\frac{1}{\Omega_{B-}^{2}-\Omega^{2}}-\frac{1}{\Omega_{B+}^{2}-\Omega^{2}}\right], \\
\varepsilon_{4}(\Omega)=\frac{q}{\left(m_{+}+m_{-}\right)}\left[\frac{\Omega_{B-}}{\Omega_{B-}^{2}-\Omega^{2}}+\frac{\Omega_{B+}}{\Omega_{B+}^{2}-\Omega^{2}}\right] .
\end{gathered}
$$

Both $\varepsilon_{3}(\Omega)$ and $\varepsilon_{4}(\Omega)$ have dimensions of an inverse frequency squared; $\varepsilon_{1}(\Omega), \varepsilon_{2}(\Omega)$ and $\varepsilon_{\|}(\Omega)$ are instead dimensionless. The frequencies appearing in Eqs. (A2)(A4) and (A5) and (A6) are the plasma and the Larmor frequencies associated with the charge carriers of both signs, i.e. $\Omega_{p \pm}=\sqrt{4 \pi q^{2} n_{0}} / m_{ \pm}$and $\Omega_{B \pm}=q B_{0} / m_{ \pm}$. To compare the dispersion relations with the standard situation of cold plasmas we must bear in mind that the ratios of the plasma and Larmor frequencies are related to the inverse ratio of the masses, i.e. $\Omega_{p+} / \Omega_{p-}=\Omega_{B+} / \Omega_{B-}=m_{-} / m_{+}$.

\section{The seven function}

The generalized form of the Appleton-Hartree equation [see Eq. (4.1)] depends on seven functions whose explicit form is given by

$$
\begin{aligned}
f_{B}(\varepsilon, \Omega, n, \theta)= & \frac{\varepsilon_{\|}}{n^{4} \Omega^{2}} \cos ^{2} \theta+\frac{\left(\varepsilon_{L}+\varepsilon_{R}\right)}{2 n^{4} \Omega^{2}} \sin ^{2} \theta \\
g_{B}(\varepsilon, \Omega, n, \theta)= & \frac{\varepsilon_{\|}\left(\varepsilon_{L}-\varepsilon_{R}\right)}{n^{5} \Omega} \cos \theta \\
f_{\omega}(\varepsilon, \Omega, n, \theta)= & \frac{\cos ^{2} \theta}{n^{4}}\left\{\left(\varepsilon_{3}-\varepsilon_{4}\right)\left(\varepsilon_{3}+\varepsilon_{4}\right) \varepsilon_{\|}+n^{2}\left[\sin \theta \varepsilon_{3}^{2}-\left(\varepsilon_{3}^{2}-2 \varepsilon_{4}^{2}\right) \sin ^{2} \theta\right]\right\}, \\
g_{\omega}(\varepsilon, \Omega, n, \theta)= & \frac{\cos \theta}{2 n^{5}}\left\{2 \varepsilon_{\|}\left[-n^{2} \varepsilon_{4}+\varepsilon_{3}\left(\varepsilon_{L}-\varepsilon_{R}\right)+\varepsilon_{4}\left(\varepsilon_{L}+\varepsilon_{R}\right)\right]-2 n^{2} \varepsilon_{4} \varepsilon_{\|} \cos ^{2} \theta+n^{2} \varepsilon_{3}\left(\varepsilon_{L}-\varepsilon_{R}\right) \sin \theta\right. \\
& \left.+n^{2}\left[4 n^{2} \varepsilon_{4}-2 \varepsilon_{3}\left(\varepsilon_{L}-\varepsilon_{R}\right)-3 \varepsilon_{4}\left(\varepsilon_{L}+\varepsilon_{R}\right)\right] \sin ^{2} \theta\right\}, \\
h_{1}(\varepsilon, \Omega, n, \theta)= & -\frac{2 \varepsilon_{4}}{n^{3} \Omega^{2}} \cos \theta \sin ^{2} \theta, \\
h_{2}(\varepsilon, \Omega, n, \theta)= & \frac{1}{2 n^{4} \Omega}\left\{4 \varepsilon_{3} \varepsilon_{\|} \cos ^{2} \theta+\sin \theta\left[\varepsilon_{3}\left(\varepsilon_{L}+\varepsilon_{R}\right)+\varepsilon_{4}\left(-\varepsilon_{L}+\varepsilon_{R}\right) \sin \theta\right]\right\}, \\
h_{3}(\varepsilon, \Omega, n, \theta)= & -\frac{\varepsilon_{3} \varepsilon_{4} \sin 2 \theta(1+\sin \theta)}{2 n^{3} \Omega} .
\end{aligned}
$$

The functions reported in Eq. (A7) determine, through Eq. (4.1), the form of the dispersion relations when the hypermagnetic and the vortical currents are simultaneously present in the anomalous magnetohydrodynamics equations.

\section{APPENDIX B: HYPERMAGNETIC KNOTS AND BELTRAMI FIELDS}

In the resistive approximation, the hyperelectric and the hypermagnetic fields are not exactly orthogonal, and the nature of this misalignment is crucial both for the generation of the baryon asymmetry and for the chiral magnetic effect. In AMHD the induced hyperelectric field stems directly from the approximate form of the Ohm's law, and it vanishes exactly, in the plasma frame, when the conductivity goes formally to infinity. ${ }^{22}$ In the same limit the contribution of the chemical potential to the anomalous hypermagnetic diffusivity equation gets always erased. At finite conductivity the anomalous contribution can be often rephrased in terms of the magnetic gyrotropy [2] which defines hypermagnetic knot solutions [10].

\footnotetext{
${ }^{22}$ In the nonrelativistic limit the conductivity $\sqrt{T / m_{-}}\left(T / \alpha^{\prime}\right)$ where $m_{-}$denotes the mass of the charge lightest charge carrier and $\alpha^{\prime}$ is the gauge coupling. If the temperature is much higher than the masses of the charge carriers the previous formula is replaced simply by $T / \alpha^{\prime}$. The perfectly conducting limit (also named infinite conducting limit) defines the ideal regime in ordinary magnetohydrodynamics. It is not uncommon, in the literature, to mention the perfectly conducting limit where the conductivity goes (formally) to infinity (see e.g. [1-3]).
} 


\section{Hypermagnetic knots}

The configurations minimizing the hypermagnetic energy density with the constraint that the helicity be conserved coincide, in the perfectly conducting limit, with the ones obtainable in ideal magnetohydrodynamics where the anomalous currents are neglected [1-4].

In the perfectly conducting limit Eq. (5.6) leads to $\partial_{t} \vec{B}=\vec{\nabla} \times(\vec{v} \times \vec{B})+\mathcal{O}\left(\bar{\mu}_{R} / \sigma\right)$ which is qualitatively similar to the result of conventional magnetohydrdynamics. Defining the vector potential in the Coulomb gauge, the magnetic diffusivity equation becomes, up to small corrections, $\partial_{t} \vec{A}=\vec{v} \times(\vec{\nabla} \times \vec{A})$. The analysis of Ref. [26] can then be exploited. The magnetic energy density is then minimized in a finite volume under the assumption of constant magnetic helicity by introducing the Lagrange multiplier $p_{B}$. By taking the functional variation of $\mathcal{G}=\int_{V} d^{3} x\left\{|\vec{\nabla} \times \vec{A}|^{2}-p_{B} \vec{A} \cdot(\vec{\nabla} \times \vec{A})\right\}$, with respect to $\vec{A}$ and by requiring $\delta \mathcal{G}=0$, the configurations extremizing $\mathcal{G}$ are such that $\vec{\nabla} \times \vec{B}=p_{B} \vec{B}$. In performing the functional variation we assumed that $V$ is the fiducial volume of a closed system.

The configurations $\vec{\nabla} \times \vec{B}=p_{B} \vec{B}$ have been used to describe hypermagnetic knots (see [10], third and fourth papers); in this case $q$ has dimensions of an inverse length and sets the scale of the hypermagnetic knot which is related to Chern-Simons waves. The configurations with constant $p_{B}$ represent the lowest state of magnetic energy which a closed system may attain also in the case where anomalous currents are present, provided the ambient plasma is perfectly conducting.

\section{Gyrotropic bases}

The knotted solutions can be expanded in an appropriate gyrotropic basis. Let us then consider a vector field $\vec{a}$ fields satisfying $\vec{a} \times(\vec{\nabla} \times \vec{a})=0$. The simplest realization of these Beltrami fields is provided by the eigenvectors of the curl operator, but more general situations are known and have been extensively examined in the literature. Two gryrotropic and orthonormal bases of opposite parity are given by $(\hat{a}, \hat{b}, \hat{z})$ and by $(\hat{c}, \hat{d}, \hat{z})$ :

$$
\begin{aligned}
& \hat{a}(z, p)=\{\cos p z,-\sin p z, 0\}, \\
& \hat{b}(z, p)=\{\sin p z, \cos p z, 0\}, \\
& \hat{c}(z, p)=\{\cos p z, \sin p z, 0\}, \\
& \hat{d}(z, p)=\{-\sin p z, \cos p z, 0\} .
\end{aligned}
$$

As anticipated the bases of Eqs. (B1) and (B2) are orthonormal. Indeed we have $\hat{a} \cdot \hat{b}=\hat{a} \cdot \hat{z}=\hat{b} \cdot \hat{z}=0$ and $(\hat{a} \times \hat{b}) \cdot \hat{z}=1$ (and similarly for $\hat{c}, \hat{d}$ and $\hat{z}$ ). The unit vectors of Eqs. (B1) and (B2) are normalized eigenvectors of the curl operator with eigenvalues $+p$ and $-p$.

In ordinary MHD knotted solutions can be constructed from Beltrami fields by postulating a solenoidal (static) current and by neglecting the displacement current. In anomalous magnetohydrodynamics these simple constructions cannot be immediately extended because of the magnetic and vortical currents. The knot solutions obtainable by extremizing the functional $\mathcal{G}$ correspond to uniform magnetic fields well inside the core of the knot. This conclusion is evident if we use the basis of Eqs. (B1) and (B2). For instance, in the limit $p z<1$ the field configuration $\vec{B}(z, p)=B_{0} \hat{a}(z, p) \rightarrow B_{0} \hat{x}$ is practically uniform and directed along the $\hat{x}$ axis. The connections between Beltrami fields, force-free solutions in ordinary MHD equilibrium and electromagnetic waves propagation have been explored in a number of papers $[18,19,26,27]$ starting from the classic works of Fermi and Chandrasekhar [28]. It is also possible to obtain hypermagnetic knot solutions with finite helicity and finite gyrotropy which do not satisfy the relation of Beltrami fields. These solutions have been studied in a number of interesting frameworks (see the last two papers of Ref. [10] and also [29,30]).
[1] H. K. Moffat, Magnetic Field Generation in Electrically Conducting Fluids, (Cambridge University Press, Cambridge, England, 1978).

[2] D. Biskamp, Magnetohydrodynamic Turbulence, (Cambridge University Press, Cambridge, England, 2003).

[3] H. Alfvén and C.-G. Fälthammer, Cosmical Electrodynamics, 2nd ed. (Clarendon Press, Oxford, 1963).

[4] E. Parker, Cosmical Magnetic Fields (Oxford University Press, Oxford, 1979).

[5] Y. B. Zeldovich, A. A. Ruzmaikin, and D. Sokoloff, Magnetic Fields in Astrophysics (Gordon and Breach, New York, 1983).
[6] T. H. Stix, Waves in Plasmas (American Institute of Physics, College Park, MD, 1992).

[7] M. Giovannini, Phys. Rev. D 88, 063536 (2013).

[8] V. A. Rubakov, Prog. Theor. Phys. 75, 366 (1986); V. A. Matveev, V. A. Rubakov, A. N. Tavkhelidze, and V.F. Tokarev, Nucl. Phys. B282, 700 (1987); V. A. Rubakov and A. N. Tavkhelidze, Phys. Lett. 165B, 109 (1985).

[9] A. N. Redlich and L. C. R. Wijewardhana, Phys. Rev. Lett. 54, 970 (1985).

[10] M. Giovannini and M. E. Shaposhnikov, Phys. Rev. D 57, 2186 (1998); Phys. Rev. Lett. 80, 22 (1998); M. Giovannini, Phys. Rev. D 61, 063502 (2000); 61, 063004 (2000). 
[11] R. D. Peccei and H. R. Quinn, Phys. Rev. Lett. 38, 1440 (1977); Phys. Rev. D 16, 1791 (1977).

[12] J. Kim, Phys. Rep. 150, 1 (1987); H.-Y. Cheng, ibid. 158, 1 (1988); G. G. Raffelt, Phys. Rep. 198, 1 (1990); Lect. Notes Phys. 741, 51 (2008).

[13] S. Carroll, G. Field, and R. Jackiw, Phys. Rev. D 41, 1231 (1990); W. D. Garretson, G. Field, and S. Carroll, Phys. Rev. D 46, 5346 (1992); G. Field and S. Carroll, Phys. Rev. D 62 , 103008 (2000).

[14] D. Kharzeev, L. McLerran, and H. Warringa, Nucl. Phys. A80, 3227 (2008); K. Fukushima, D. Kharzeev, and H. Warringa, Phys. Rev. D 78, 074033 (2008); D. Kharzeev, Ann. Phys. (Amsterdam) 325, 205 (2010).

[15] K. Landsteiner, E. Megias, L. Melgar, and F. Pena-Benitez, Fortschr. Phys. 60, 1064 (2012); J. High Energy Phys. 09 (2011) 121; V. A. Rubakov, arXiv:1005.1888; T. Kalaydzhyan and I. Kirsch, Phys. Rev. Lett. 106, 211601 (2011); I. Gahramanov, T. Kalaydzhyan, and I. Kirsch, Phys. Rev. D 85, 126013 (2012); V. P. Nair, R. Ray, and S. Roy, Phys. Rev. D 86, 025012 (2012).

[16] D. T. Son and P. Surowka, Phys. Rev. Lett. 103, 191601 (2009).

[17] H. L. Pécseli, Waves and Oscillations in Plasmas (CRC Press, Boca Raton, FL, 2012).

[18] C. Chu and T. Ohkawa, Phys. Rev. Lett. 48, 837 (1982).

[19] N. Salingaros, Phys. Rev. A 35, 4856 (1986); 45, 8811 (1992); 45, 8816 (1992).

[20] J. Ahonen, Phys. Rev. D 59, 023004 (1998); J. Ahonen and K. Enqvist, Phys. Lett. B 382, 40 (1996); H. Heiselberg, Phys. Rev. D 49, 4739 (1994).
[21] Y. Hirono, M. Hongo, and T. Hirano, Phys. Rev. C 90, 021903 (2014); Y. Yin, Phys. Rev. C 90, 044903 (2014).

[22] N. Yamamoto, Phys. Rev. Lett. 115, 141601 (2015).

[23] G. Piccinelli and A. Ayala, Lect. Notes Phys. 646 (2004); K. Bamba, Phys. Rev. D 74, 123504 (2006); L. Campanelli and M. Giannotti, Phys. Rev. Lett. 96, 161302 (2006).

[24] K. Bamba, C. Q. Geng, and S. H. Ho, Phys. Lett. B 664, 154 (2008); L. Campanelli, Int. J. Mod. Phys. D 18, 1395 (2009); Eur. Phys. J. C 74, 2690 (2014); S. Ozonder, Phys. Rev. C 81, 062201 (2010); 84, 019903 (2011).

[25] B. A. Fayzullaev, M. M. Musakhanov, D. G. Pak, and M. Siddikov, Phys. Lett. B 609, 442 (2005); M. N. Chernodub and A.J. Niemi, Phys. Rev. D 79, 077901 (2009); P. M. Akhmet'ev, V. B. Semikoz, and D. D. Sokoloff, Pis'ma Zh. Eksp. Teor. Fiz. 91, 233 (2010) [JETP Lett. 91, 215 (2010)].

[26] S. Chandrasekhar and L. Woltjer, Proc. Natl. Acad. Sci. U.S.A. 44, 285 (1958); L. Woltjer, Proc. Natl. Acad. Sci. U.S.A. 44, 489 (1958).

[27] S. Chandrasekhar and P. C. Kendall, Astrophys. J. 126, 457 (1957).

[28] E. Fermi and S. Chandrasekhar, Astrophys. J. 118, 116 (1953).

[29] C. Adam, B. Muratori, and C. Nash, Phys. Rev. D 61, 105018 (2000); R. Jackiw and S. Y. Pi, Phys. Rev. D 61, 105015 (2000).

[30] C. Adam, B. Muratori, and C. Nash, Phys. Rev. D 62, 105027 (2000); Phys. Lett. B 485, 314 (2000). 